\title{
A Novel PM2.5 Concentrations Probability Density Prediction Model Combines The Least Absolute Shrinkage And Selection Operator With Quantile Regression
}

\section{Shaomei Yang}

North China Electric Power University - Baoding Campus

Haoyue Wu ( $D$ whylikefreshair@126.com )

North China Electric Power University - Baoding Campus https://orcid.org/0000-0002-3230-6288

\section{Research Article}

Keywords: PM2.5 concentration prediction, probability density prediction, the least absolute shrinkage and selection operator, quantile regression, Air pollution, Hybrid models

Posted Date: November 22nd, 2021

DOl: https://doi.org/10.21203/rs.3.rs-982230/v1

License: (c) (1) This work is licensed under a Creative Commons Attribution 4.0 International License.

Read Full License 
A novel PM2.5 concentrations probability density prediction model combines the least absolute shrinkage and selection operator with quantile regression

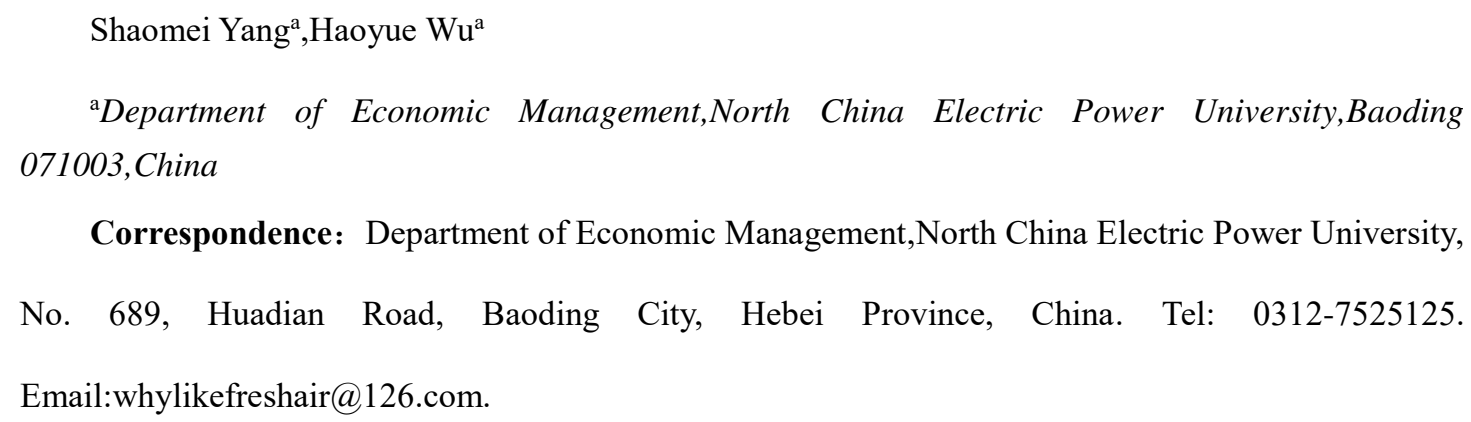

Correspondence: Department of Economic Management,North China Electric Power University, No. 689, Huadian Road, Baoding City, Hebei Province, China. Tel: 0312-7525125. Email:whylikefreshair@126.com.

Abstract: PM2.5 has a significant negative impact on human health and atmospheric quality, and accurate prediction of its concentration is necessary. PM2.5 concentration is influenced by a combination of factors from both meteorological conditions and air quality. It is essential to identify the significant factors influencing PM2.5 concentrations in the prediction process. To address this issue, this paper proposes the quantile regression $(\mathrm{QR})$ model based on the least absolute shrinkage and selection operator (LASSO), combined with kernel density estimation (KDE) for probabilistic density prediction of PM2.5 concentrations. The model uses LASSO regression to select the influential factors, and then the quartiles of daily PM2.5 concentrations obtained using the QR model are imported into the KDE model to obtain the probability density curves of PM2.5 concentrations. In this paper, empirical analysis is performed with the data sets of Beijing, China, and Jinan, China, and the accuracy of the model is evaluated using the mean absolute percentage error(MAPE) and the relative mean square error (RMSE). The simulation results reveal that the LASSSO-QR-KDE model has a higher accuracy than the traditional prediction models and the currently used research models. The model provides a novel and excellent tool for policy makers to predict PM2.5 concentrations.

Keywords: PM2.5 concentration prediction; probability density prediction; the least absolute 
shrinkage and selection operator; quantile regression; Air pollution; Hybrid models

\section{Introduction}

PM2.5 refers to the particulate matter in the ambient air with an aerodynamic equivalent diameter less than or equal to 2.5 microns, which has a small particle size and can be used as a carrier of toxic substances. These fine particles can be inhaled into the human body through the respiratory system and adhere to the upper and lower respiratory tracts and lung lobes, causing damage to the nervous system, cardiovascular and cerebrovascular systems, and respiratory systems.People who have been exposed to fine particulate pollution for a long time are more likely to face cardiovascular and respiratory diseases (Geng et al. 2015; Lee et al. 2017; Mukhopadhyay and Sahu 2018). At the same time, PM2.5 pollution has a negative impact on the normal development of agriculture, industry, and cultural tourism. It will also affect traffic order and cause major economic losses.By predicting the concentration of PM2.5 and accurately forecasting it, it is possible to issue early warnings for heavily polluted weather in order to take targeted protective measures. Therefore, in order to maintain the order of production activities, reduce public health risks, and provide meaningful reference for relevant policy makers, the prediction of PM2.5 concentration is of great significance.

Currently, prediction models for air pollutant concentrations such as PM2.5 can be divided into three main categories:deterministic models, classical statistical models, and machine learning models (Jiang and Qiao 2021). Deterministic methods mainly include weather research forecasting model (WRF) (Cao et al. 2018), community multiscale air quality model (CMAQ) (Yang et al. 2019), and coupled WRF-CMAQ meteorological-chemical model (Syrakov et al. 2016). The above methods use relevant meteorological data and pollutant source data to simulate the process of pollutants from 
emission to accumulation to dispersion and transfer, so as to predict and analyze the concentration of pollutants.According to Lightstone et al. (2017), such methods are more complex and do not have a significant advantage in prediction accuracy over other models. At the level of statistical models, autoregressive integrated moving average model (ARIMA) and multiple linear regression model (MLR) are more used among scholars in the problem of PM2.5 concentration prediction (Zhang et al. 2018).However, the concentration of PM2.5 is affected by a variety of variable factors and has strong nonlinearity and complexity. Statistical models are not satisfactory in processing nonlinear time series data. Machine learning has the advantage of covering knowledge of probability theory, statistics, approximate theory and knowledge of complex algorithms, and can simulate the way of human learning. Machine learning has superior predictive performance and is widely used in air pollutant concentration prediction.At the same time, the hybrid model is also widely used in the prediction of the concentration of air pollutants such as PM2.5. Huang and Kuo (2018) combined convolutional neural network (CNN) and long short-term memory (LSTM) and applied it to the PM2.5 prediction system. This method effectively improved the prediction accuracy of the LSTM model.Huang et al. (2021) used empirical mode decomposition (EMD) to decompose the PM2.5 concentration series. They input the multiple stationary sub-sequences and meteorological features obtained after decomposition into the constructed gated recurrent unit neural network(GRU) in order to train and predict them. The results showed that the accuracy of the EMD-GRU model is much higher than that of the single GRU model.Li et al. (2017) introduced the co-integration theory in order to solve the problem of the lack of evaluation of the possible correlation between different variables in the current research. He used the Flower Pollination Algorithm (FPA) to optimize the parameters of the Support Vector Machine (SVM) model, and made high-precision predictions of the PM2.5 and PM10 concentrations in two important 
cities in Yunnan Province, China.

The concentration of PM2.5 is affected by many factors, not only related to meteorological factors, but also closely related to economic development mode, industrial structure, and energy efficiency. In the big data environment, related data sources are extensive and the characteristics are different, making the prediction of PM2.5 concentration more complicated.Reviewing the existing literature related to the prediction of PM2.5 and other air pollutant concentrations, it is found that most of the prediction methods obtain deterministic point prediction results, which can hardly reflect the uncertainty of their fluctuations. To solve the above problems, a probability density prediction method for PM2.5 concentration can be used to overcome the shortcomings of the traditional point prediction and interval prediction methods. In this way, the future PM2.5 concentration values and the concentration fluctuation intervals under the corresponding confidence intervals can be obtained, and the probability of occurrence of each point within the interval can also be visualized. With the probability density function of the predicted PM2.5 concentration, more detailed and effective information can be obtained than the traditional prediction methods. It is extremely helpful for the accurate prediction of PM2.5 concentration.

However, due to the complexity and variability of factors affecting PM2.5 concentration, the probability density function that accurately portrays the uncertainty of PM2.5 concentration is difficult to obtain. Therefore, in order to predict PM2.5 concentration more accurately, valuable information needs to be extracted from the wide range of influencing factors first, so as to filter out the influencing factors that have significant explanatory significance on PM2.5 concentration. The traditional classical method of variable selection is mainly represented by the subset selection method. This method compared $2^{p}-1$ non-empty subsets of all p-dimensional variables with a certain criterion to find the 
optimal subset. Here the most representative selection criteria are mainly the Akaike information criterion (AIC) based on information theory proposed by Hirotsugu Akaik in 1974 (Hoerl and Kennard 2000), the BIC criterion based on Bayes' method proposed by Schwarz (1978), and the cross-validation criterion. But for the information age where high-dimensional data are proliferating in various fields, the complexity and high cost of computation make the traditional variable selection methods no longer applicable to these high-dimensional data structures. Therefore, statisticians have started to focus on variable selection methods for high-dimensional data. In the field of variable selection for high-dimensional data, Tibshirani's 1996 proposal of the LASSO algorithm (Least Absolute Shrinkage and Selection Operator) under the ordinary linear model was of epoch-making significance (Tibshirani 1996). It was inspired by two algorithms, ridge Regressionl (Hoerl and Kennard 2000) and Nonnegative Garrote (Breiman 1995).

LASSO was essentially a coefficient compression method that compressed the coefficients of non-significantly influential variables to zero, achieving both variable selection and parameter estimation at the same time. This method can be seen as an improvement of ridge regression, but it is milder in compressing the coefficients of significant variables with significant effects, thus ensuring the accuracy of parameter estimation. In a previous study, Koen et al. (2021) used a LASSO regression model to select variables closely related to four common chronic diseases in the Netherlands to predict disease prevalence. The results showed that the use of the LASSO algorithm significantly improved the predictive performance of the model. Jasleen and Mamta (2021) used a correlation-based adaptive LASSO algorithm to identify potentially important influences while studying pollutant concentrations and meteorological factors in Delhi and its surrounding cities. Evaluation of the model revealed better performance of this approach in extracting subset of features. In an empirical study, Li et al. (2021) 
found that real surplus management (REM) was strongly selected as a key crisis predictor by the LASSO algorithm, developing an updated distress forecasting model with surplus management considerations for the Chinese market. In addition, the method has been applied to the variable selection process of quantile regression models.

Quantile regression was first proposed by Koenker and Bassett (1978). Due to its unique explanatory power, it has become one of the key tools for data analysis and is widely used in various data analysis fields (Koenker 2015). Quantile regression can give a more comprehensive picture of the conditional distribution of the explanatory variables. Instead of analyzing only the conditional expectation (mean) of the explanatory variable, the method can also analyze how the explanatory variables affect the median, quantile, etc. of the explanatory variable. Kang et al. (2021) used quantile regression to study the impact of macroprudential policies on the level of bank financing of Chinese firms, and pointed out that the intensity of the impact of macroprudential policies was different at different quantile points. salari et al. (2021) combined the idea of quantile regression in the study of the impact of multiple factors on the ecological footprint of emerging countries, and discussed the significant impact of different quantile factors. Sun et al. (2021) used quantile autoregressive models to analyze return and volatility series on multiple time scales using the crude oil market as an example. It was found that the autoregressive coefficients vary with quantile. The above study further demonstrates the advantages of quantile regression. Namely, the method can explain the effect of each input variable on the corresponding variable at different quartiles and obtain a more detailed relationship between the input and response variables.

The approach of combining the LASSO algorithm with quantile regression has been applied to the power, financial, and medical sectors. he et al. (2019) predicted electricity consumption based on 
133

LASSO-quantile regression for Guangdong Province, China, and California, USA. Simulation results showed that the method provided better performance for electricity consumption forecasting. Cao et al. (2021) studied the optimal portfolio problem based on the LASSO-quantile regression method and showed that the results obtained by this method were close to the given value at risk under certain canonical conditions, demonstrating the robustness and effectiveness of this method.Taha et al. (2018) reduced the data dimensionality by LASSO and used quantile regression method to study and analyze the prostate cancer dataset. The results validated the superiority of this combined method.

There are no studies that have applied LASSO quantile regression to air pollutant concentration prediction. In this paper, the LASSO-QR-KDE model is proposed to combine LASSO quantile regression with kernel density estimation. Firstly, this paper will apply the LASSO variable selection method to extract the factors that have significant effects on PM2.5 concentrations from the high-dimensional external influences data to obtain a lower dimensional data set. Subsequently, the quantile regression model is combined with the quantile regression method to obtain the conditional quantile of PM2.5 concentrations at different quantile points. Finally, the kernel density estimation method was employed to obtain the probability density prediction curves of PM2.5 concentrations on any consecutive day in the future month.

The main innovations and contributions of this paper can be divided into the following two aspects:

(1) The LASSO-QR-KDE model proposed in the paper reduces the uncertainty of the prediction by downscaling the influencing factors of PM2.5 concentration and selecting the factors that have a significant influence on it. Moreover, it is able to obtain different probability distributions of PM2.5 
concentrations at different quantile points and further obtain probability density curves. Compared with the common point prediction, the probability density prediction expands the prediction range and provides a more detailed and informative explanation for the prediction of PM2.5 concentrations.

(2) This paper demonstrates the superiority of the method through experiments on two real data sets in Beijing and Jinan, China. Three different scenarios are investigated and compared in this paper: without considering external factors, considering external factors without variable selection, and using the LASSO algorithm to filter external factors. The results show that the proposed LASSO-QR-KDE model has superior performance, and its MAPE and RMSE are both taken to the minimum value. Moreover, when comparing five models commonly used to predict PM2.5 concentrations, namely ARIMA, BP, RBF, CNN-LSTM and EMD-GRU, the LASSO-QR-KDE model was found to achieve higher accuracy.

The rest of the paper is organized as follows: the second part describes the theoretical basis and methodology involved in the model proposed in the paper. The third part presents the constructed neural network model and its evaluation criteria. The fourth part gives an empirical analysis and discussion of the two cases and shows how the data are handled. Finally, the conclusions of the paper are given in the fifth part.

\section{Methodology}

\subsection{LASSO method}

The LASSO method adds a penalty term to optimize the traditional objective function to produce a sparse solution. The method avoids the problem of overfitting caused by excessive explanatory variables (He et al. 2021). It proceeds by extracting the key variables from the high-dimensional 
175

variables. The method is effectively solving the problem of multicollinearity among variables and improving the explanatory accuracy of the model, which is highly evaluated and widely used. In addition, compared with ridge regression, the Lasso method can directly compress the regression coefficients of redundant predictor variables to zero, obtaining a more streamlined set of predictor variables. Over-compression of important regression coefficients can also be avoided.

Given a matrix of independent variables $X=\left(x_{1}, x_{2}, \cdots, x_{n}\right)$, where $X_{j}=\left(x_{1 j}, x_{2 j}, \cdots, x_{m j}\right)^{T}$, $j=1,2, \cdots, n$, the dependent variable $Y=\left(y_{1}, y_{2}, \cdots, y_{m}\right)^{T}$. It is assumed that the data have been standardized, $\sum_{i=1}^{m} x_{i j}=0, \sum_{i=1}^{m} x_{i j}^{2}=1, \sum_{i=1}^{m} y_{1}=0$, then the linear model between $Y$ and $X$ is established as Eq. (1).

disturbance term.

Suppose $\beta=\left(\beta_{1}, \beta_{2}, \cdots, \beta_{n}\right)^{T}$, then the LASSO estimation of $\beta$ is as in Eq. (2).

$$
\hat{\beta}^{\text {LASSO }}=\operatorname{argmin} \sum_{i=1}^{m}\left(y_{i}-\beta_{0}-\sum_{j=1}^{n} x_{i j} \beta_{j}\right)^{2}
$$

$$
\text { s.t. } \quad \sum_{j=1}^{n}\left|\beta_{j}\right| \leq \lambda
$$

where the penalty parameter is $\lambda$ and $\lambda \geq 0$. If $\hat{\beta}^{0}$ is the least squares estimation of $\beta_{j}$, then $\lambda_{0}=\sum_{j=1}^{n}\left|\hat{\beta}^{0}\right|$. When $\lambda<\lambda_{0}$, the absolute value of the LASSO estimation of the regression coefficient $\beta_{j}$ in the model is smaller than the absolute value of $\hat{\beta}^{0}$. As $\lambda$ decreases gradually, the LASSO estimation of certain $\beta_{j}$ decreases or even goes to zero. The variable can be eliminated if its estimation is zero, which indicates that it has little association with the dependent variable $Y$, and thus 
195

196

197

the variable selection function can be achieved. The commonly used estimation and testing methods regarding the penalty parameter $\lambda$ are the cross-validation criterion, the generalized validation method, and the AIC (Hoerl and Kennard 2000).

For the solution of the LASSO problem, the least angle regression (LARS) significantly enhances the computational efficiency of LASSO.Suppose $A=\left\{j_{1}, j_{2}, \cdots, j_{k}\right\} \subseteq\{1,2, \cdots, n\}$ is the indicator set, and define the matrix of $m \times k$ as $X_{A}=\left(\lambda_{j 1} x_{j 1}, \lambda_{j 2} x_{j 2}, \cdots, \lambda_{j k} x_{j k}\right)$, where the value of $\lambda$ can be determined by Eq. (3).

$$
\lambda_{j l}\left\{\begin{array}{c}
1, x_{j l} \text { is positively correlated with } Y \\
0, x_{j l} \text { is not correlated with } Y \\
-1, \quad x_{j l} \text { is negatively correlated with } Y
\end{array}\right.
$$

Subsequently, suppose that $G_{A}=X_{A}^{\prime} X_{A}$ and $A_{A}=\left(I_{A}^{\prime} G_{A}^{-1} I_{A}\right)^{-\frac{1}{2}}, u_{A}=X_{A} w_{A}$ is the angular component vector in $X_{A}, w_{A}=A_{A} G_{A}^{-1} I_{A}$. where $I_{A}$ is a $\mathrm{K}$-dimensional column vector and all elements are equal to 1 . It can be shown that the angular component vector $u_{A}$ is a unit vector and $X_{A}^{\prime} u_{A}=A_{A} I_{A}$.

Establish the LARS estimation as $\hat{\mu}=X \hat{\beta}$. Assuming that the current estimate is $\hat{\mu}_{A}, \hat{c}=$ $X^{\prime}\left(Y-u_{A}\right)$ indicates that the respective variable is currently correlated with $Y$. From the previous assumptions, the variables in $A$ are maximally correlated with $Y$, and the relationship can be expressed by Eq. (4).

$$
\left\{\begin{array}{l}
\left|\hat{c}_{j}\right|=\widehat{C}, j \in A \\
\left|\hat{c}_{j}\right|<\widehat{C}, j \notin A
\end{array}\right.
$$

where $\widehat{C}=\max _{j}\left\{\left|\hat{c}_{j}\right|\right\}$.

(1)Calculate the value of $\widehat{C}$.

(2)Calculate $X_{A}, A_{A}, u_{A}$ by taking $\lambda_{j}=\operatorname{sign}\left\{\hat{c}_{j}\right\}(j \in A)$. 
(4)Calculate $\hat{\mu}_{A+}=u_{A}+\hat{\gamma} u_{A}$, where $\hat{\gamma}=\min _{j \notin A}+\left\{\frac{\widehat{C}-\hat{c}_{j}}{A_{A}-a_{j}}+\frac{C+c_{j}}{A_{A}+a_{j}}\right\} . \min ^{+}$means that only the smallest positive value in the set is selected for calculation. variables are used for the operation.

\subsection{Quantile regression}

$$
Q(\tau \mid X)=\alpha_{0}(\tau)+\alpha_{1}(\tau) X_{1}+\alpha_{2}(\tau) X_{2}+\cdots+\alpha_{m}(\tau) X_{m}=X^{\prime} \alpha(\tau)
$$

where $Q(\tau \mid X)$ is the estimate of the response variable $Y$ corresponding to the explanatory variable $X=\left[X_{1}, X_{2}, \cdots, X_{m}\right]$ under the $\tau$ th quantile condition. $\alpha(\tau)=\left[\alpha_{0}(\tau), \alpha_{1}(\tau), \cdots, \alpha_{m}(\tau)\right]^{T}$ is the model parameter associated with the quantile $\tau$, which is estimated and solved by Eq. (6).

$$
\begin{gathered}
\min \sum_{i=1}^{N} \rho_{\tau}\left(Y_{i}-X_{i} \alpha(\tau)\right) \\
=\min \left[\sum_{i \mid Y_{i} \geq X_{i} \alpha(\tau)} \tau\left(Y_{i}-X_{i} \alpha(\tau)\right)+\sum_{i \mid Y_{i}<X_{i} \alpha(\tau)}(\tau-1)\left(Y_{i}-X_{i} \alpha(\tau)\right)\right]
\end{gathered}
$$
solved according to Eq. (5). 
The solution of the density function of a random variable can be solved by parametric estimation methods and nonparametric estimation methods. However, the parametric estimation method requires prior estimation of the density function form of the random variable, which often leads to a large discrepancy between the actual density function and the estimated density function. estimation methods.KDE is used in probability theory to estimate unknown density functions and was proposed by Rosenblatt and Emanuel Parzen (1991). The method enables to employ a set of random variables from the same unknown distribution function to estimate its density function. KDE has no requirement to know the prior distribution of the data in question or to make any assumptions about the data in question. The method requires only input variables, kernel density estimation function and optimal bandwidth to perform the predictions. of KDE, a simple estimate of $f(x)$ can be obtained as in Eq. (8).

$$
f(x)=\frac{F(x+k)-F(x-k)}{2 k}
$$

where $k$ is a non-negative series of constants and $F(x)$ is the sample distribution function of the random variable $x . x_{1}, x_{2}, \cdots, x_{n}$ are $n$ sample points of an independent identically distributed

251 one-dimensional continuous overall sample. Its probability density function is $f(x)$ and the kernel density estimate is as in Eq. (9).

$$
\hat{f}_{h}(x)=\frac{1}{n} \sum_{i=1}^{n} K_{h}\left(x-x_{i}\right)=\frac{1}{n h} \sum_{i=1}^{n} K\left(\frac{x-x_{i}}{h}\right)
$$


where $K(\cdot)$ is the kernel function, which is essentially the weight function. $h$ is called the bandwidth, and its choice determines the smoothness and accuracy of the estimated density function.

\section{LASSO-QR-KDE model}

\subsection{Quantile regression model based on LASSO}

The precision of PM2.5 concentration prediction is influenced by various external factors, and the impact of different factors on the prediction results varies in degrees. According to the description of quantile regression and the LASSO method in the previous section, the quantile regression model is constructed as Eq. (10).

$$
Y_{t}=r(\tau)+\sum_{c=1}^{p} \alpha_{c}(\tau) Y_{t-c}+\sum_{d=1}^{m}\left(\sum_{j=1}^{q} \beta_{j}^{d}(\tau) X_{t-j}^{d}\right)+\varepsilon_{t}
$$

where $Y_{t}$ is the prediction series and $X_{t}$ is the sequence of influencing factors. $m$ indicates the number of influencing factors and $c$ indicates the maximum number of lags of the prediction series, $c=1,2, \cdots, p . \tau$ is the quantile, $\tau \in(0,1)$. The regression coefficient vector for the prediction target of the $\tau$ th quantile is $\alpha_{c}(\tau)$, and the regression coefficient vector for the $\tau$ th quantile is $\beta_{j}^{d}(\tau), d=$ $1,2, \cdots, m . q$ indicates the maximum number of lags of the influence factor. $r(\tau)$ is the intercept term and $\varepsilon_{t}$ is the error term. The predicted value of PM2.5 concentration $y_{t}$ at the $\tau$ th quantile can be further obtained, which is calculated as Eq. (11)

$$
Q_{Y_{t}}\left(\tau \mid Y_{t}, X_{t}\right)=r(\tau)+\sum_{c=1}^{p} \alpha_{c}(\tau) Y_{t-c}+\sum_{d=1}^{m}\left(\sum_{j=1}^{q} \beta_{j}^{d}(\tau) X_{t-j}^{d}\right)
$$

According to Eqs. (10) and (11), it can be observed that there is heterogeneity in $r(\tau), \alpha_{c}(\tau)$, and $\beta_{j}^{d}(\tau)$, which indicates the discrepancy in the importance of the studied samples. This reflects the impact of the selected influence factors on the PM2.5 concentration prediction results under different 
quantile points. The parameters $\alpha_{c}(\tau)$ and $\beta_{j}^{d}(\tau)$ in Eq. (11) can be solved by Eq. (12).

$$
\min \sum_{t=1}^{n} \rho_{\tau}\left(Y_{t}-r(\tau)-\sum_{c=1}^{p} \alpha_{c}(\tau) Y_{t-c}-\sum_{d=1}^{m}\left(\sum_{j=1}^{q} \beta_{j}^{d}(\tau) X_{t-j}^{d}\right)\right)
$$

where $\rho_{\tau}(u)$ is the check function with the following Eq. (13).

$$
\rho_{\tau}(u)=(\tau-I(u<0)) u
$$

$I(u<0)$ is called the indicator function and $u<0$ is the conditional relation. When $u<0$ is true, $I(u<0)=1$, and vice versa $I(u<0)=1$. To improve the speed of operation, the interior point algorithm is applied to solve the equation (Portnoy and Koenker 1997).

Since the influence of multiple factors on PM2.5 concentration is considered, this paper will apply the LASSO method to filter a large number of influence factors. The penalty parameter L1 is added to the original objective function, and Eq. (14) is obtained as follows.

$$
\begin{gathered}
\min \sum_{t=1}^{n} \rho_{\tau}\left(Y_{t}-r(\tau)-\sum_{c=1}^{p} \alpha_{c}(\tau) Y_{t-c}-\sum_{d=1}^{m}\left(\sum_{j=1}^{q} \beta_{j}^{d}(\tau) X_{t-j}^{d}\right)\right) \\
+\lambda\left(\|\alpha(\tau)\|_{1}+\|\beta(\tau)\|_{1}\right)
\end{gathered}
$$

where $\lambda$ is the penalty parameter and its penalty enhances as the value taken increases. $\|\alpha(\tau)\|_{1}$ and $\|\beta(\tau)\|_{1}$ are the 1-norm. $\|\alpha(\tau)\|_{1}$ is the loss function that measures the effectiveness of the quantile regression model to fit the data. $\|\beta(\tau)\|_{1}$ is the penalty function, which can be used to achieve variable selection by constraining the insignificant coefficients to zero. To facilitate the solution of the LASSO-QR model, Eq. (14) can be transformed into Eq. (15). 


$$
\min \sum_{t=1}^{n} \rho_{\tau}\left(Y_{t}-r(\tau)-\sum_{c=1}^{p} \alpha_{c}(\tau) Y_{t-c}-\sum_{d=1}^{m}\left(\sum_{j=1}^{q} \beta_{j}^{d}(\tau) X_{t-j}^{d}\right)\right)
$$

$$
\text { s.t. } \quad\|\alpha(\tau)\|_{1}+\|\beta(\tau)\|_{1}<\delta
$$

\subsection{Probability density prediction of PM2.5 concentration} follows.

$$
k(x)=\frac{3}{4}\left(M-x^{2}\right) M \quad(|x| \leq 1)
$$


Neural networks employ nonlinear kernel functions that can provide a better way to deal with complex nonlinear objects. In the paper, a quantile regression neural network is used to predict the quantile corresponding to random variables at different quantile levels based on the single hidden layer neural network model proposed by Taylor (Madsen and Nielsen 1993). The hyperbolic tangent Sigmoid function is used as the hidden layer basis function of the neural network in solving the problem utilizing quantile regression neural network. First a stable network structure is established between the input and response variables to achieve the prediction of different quartiles of the predicted object, and then the probability density can be estimated by using the kernel density estimation of nonparametric estimation. The expression of the quantile regression neural network model used in the paper is as follows.

$$
Q_{Y}(\theta \mid X)=f[x, u(\theta), v(\theta)]=\sum_{j=1}^{J}\left\{\frac{2 v_{j}(\theta)}{1+e^{-2 \sum_{i=1}^{n} u_{i j}(\theta) X_{i}}}-v_{j}(\theta)\right\}
$$
penalty term is added to the objective function function. The new objective function is as follows

$$
E_{\theta}(U(\theta), V(\theta))=\tilde{E}_{\theta}+\lambda_{1} \sum_{i, j} u_{i j}(\theta)+\lambda_{2} \sum_{i, j} v_{j}(\theta)
$$


parameters, the model can be effectively prevented from over-fitting to the empirical data, which can

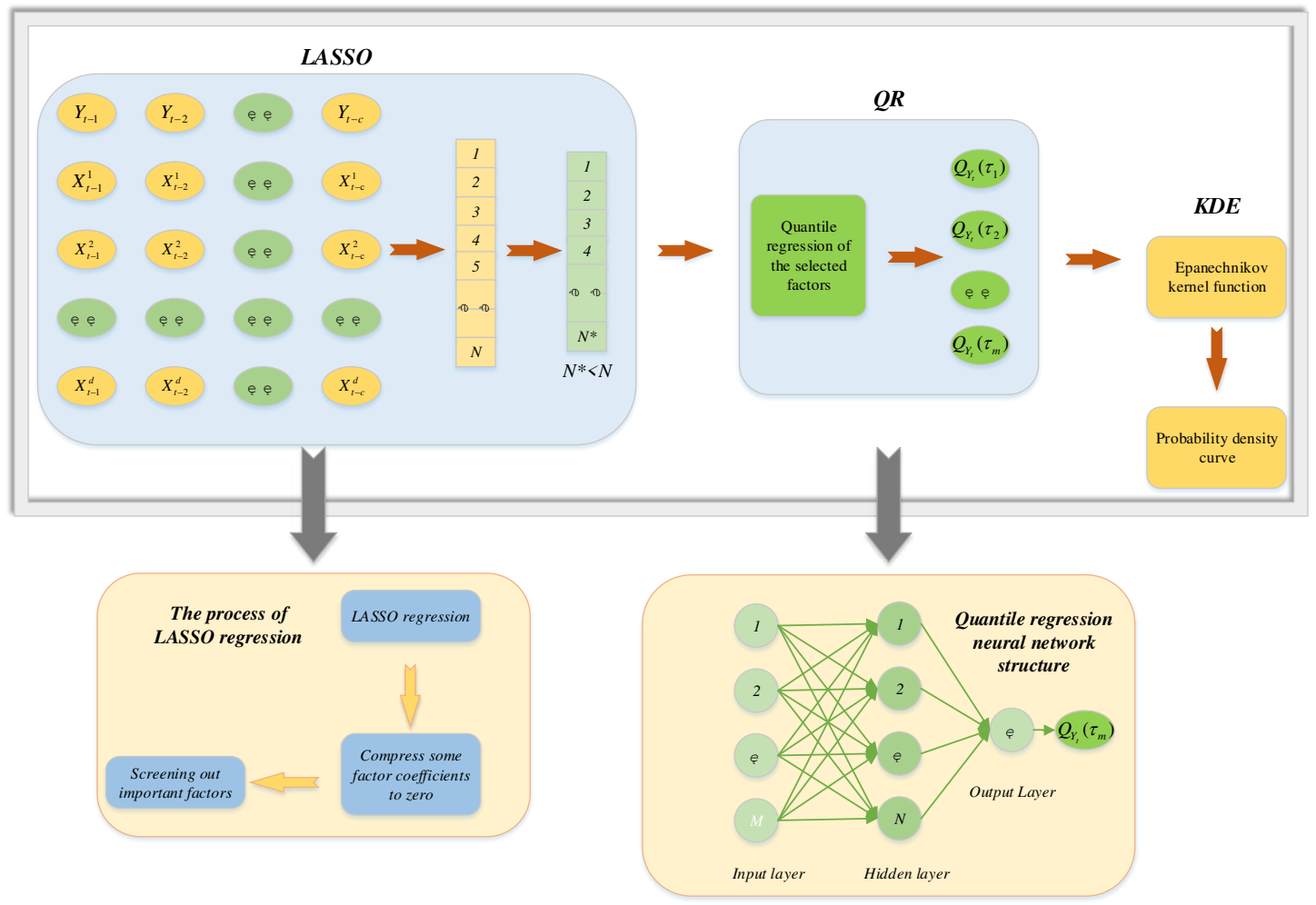

Figure.1. Flow chart of the model 
sensitive to outlier data and can highlight error values with large effects, and the expressions are as follows.

$M A P E=\frac{1}{n} \sum_{i=1}^{n}\left|\frac{A_{i}-F_{i}}{A_{i}}\right|$
$R M S E=\sqrt{\frac{\sum_{i=1}^{n}\left(A_{i}-F_{i}\right)^{2}}{n}}$

where $n$ indicates the number of days of PM2.5 concentration being used for prediction, $A_{i}$ is the actual value, and $F_{i}$ is the predicted value. The closer the values of MAPE and RMSE to zero indicates the better model performance.

\section{Empirical analysis}

\subsection{Case 1: Beijing, China}

Beijing is located in the north of China and has 12 national automatic air quality monitoring sites.

21 century, Beijing has developed rapidly and made great achievements in industrial construction. However, along with the economic boom, the air quality in Beijing has also been affected, and it is far from the national standard and the expectation of the people, especially the foggy weather makes the whole society pay extra attention to the fine particulate matter PM2.5 pollution which is more difficult to prevent and control. In recent years, Beijing has vigorously promoted clean energy and strengthened environmental supervision, enabling pollutant concentrations to drop significantly. However, the first quarter still has a high PM2.5 concentration, as shown in Fig.2. In order to continuously promote the environmental construction, the prediction of PM2.5 is essential.

In this case, the data were selected from January 1, 2018 to January 31, 2019 for Beijing, China. The data were obtained from the National Meteorological Data Center of China (http://data.cma.cn/), 
and all data were normalized in advance. From the experience of previous studies, the concentration of PM2.5 is influenced not only by air quality but also by meteorological factors. In this paper, five air quality indicators and five meteorological factor indicators were selected as influencing factors to investigate the implications on PM2.5 concentration prediction.

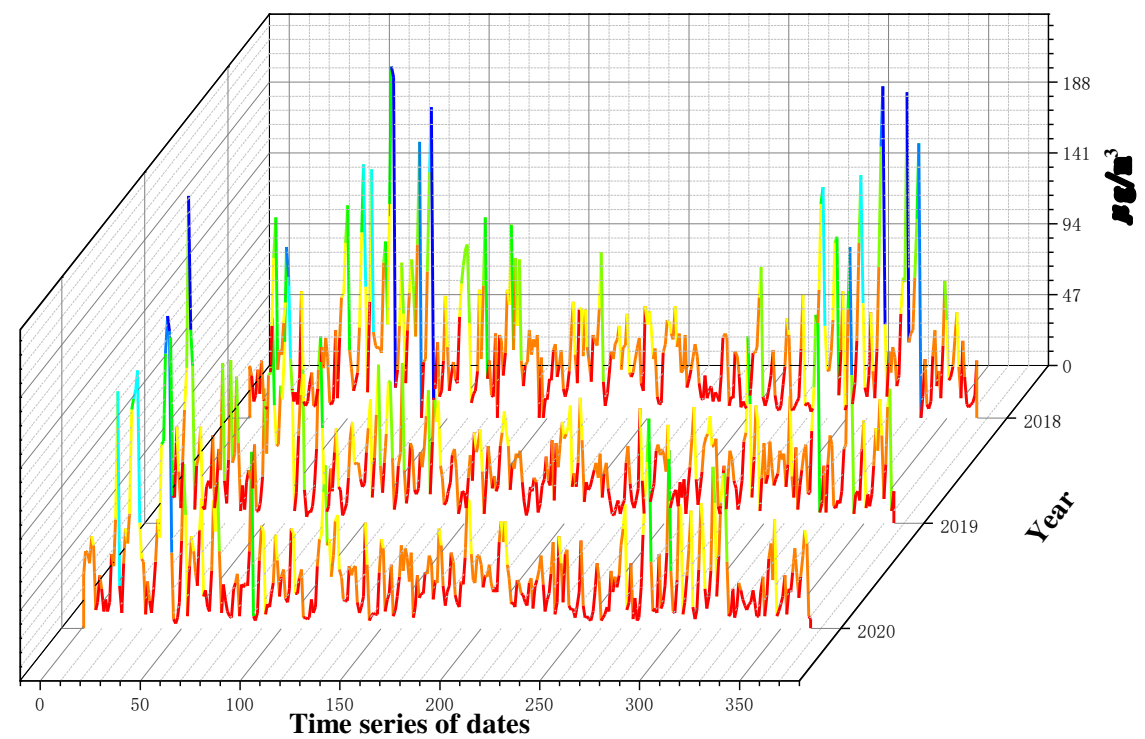

Fig.2. The PM2.5 concentration in Beijing from 2018 to 2020

According to the previous Eq. (11), the sequence of PM2.5 concentrations to be predicted is $\left(\mathrm{y}_{1}, \mathrm{y}_{2}, \cdots, \mathrm{y}_{\mathrm{n}}\right)$, where $\mathrm{n}$ is the number of samples to be predicted. The sequence of influencing factors is $X_{\mathrm{t}}$ and $X_{t}=\left(X_{t}^{1}, X_{t}^{2}, \cdots, X_{t}^{m}\right), \mathrm{m}$ is taken as 10 in this paper. $\mathrm{X}_{\mathrm{t}}^{\mathrm{d}}$ is the $d$ th influencing factor, and $X_{t}^{d}=\left(x_{t-1}^{d}, x_{t-2}^{d}, \cdots, x_{t-q}^{d}\right)$. In this paper, $X_{t}^{1}$ indicates daily PM10 concentration $(\mu \mathrm{g} / \mathrm{m} 3), X_{t}^{2}$ indicates daily $\mathrm{SO} 2$ concentration $(\mu \mathrm{g} / \mathrm{m} 3), X_{t}^{3}$ indicates daily $\mathrm{CO}$ concentration $(\mathrm{mg} / \mathrm{m} 3), X_{t}^{4}$ indicates daily NO2 concentration $(\mu \mathrm{g} / \mathrm{m} 3)$, and $X_{t}^{5}$ indicates daily O3 concentration $(\mu \mathrm{g} / \mathrm{m} 3) ; X_{t}^{6}$ indicates daily average temperature $\left(0.1^{\circ} \mathrm{C}\right), X_{t}^{7}$ indicates the daily relative humidity $(\%), X_{t}^{8}$ indicates the daily average air pressure $(0.1 \mathrm{hPa}), X_{t}^{9}$ indicates the daily average wind speed $(0.1 \mathrm{~m} / \mathrm{s})$, and $X_{t}^{10}$ indicates the wind direction of daily maximum wind speed (16 directions). The details of each 
influencing factor are shown in Table 1. $Y_{\mathrm{t}}=\left(y_{\mathrm{t}-1}, y_{\mathrm{t}-2}, \cdots, y_{t-c}\right)$ is used to indicate the historical PM2.5 concentration with lag $c$ period. Both $c$ and $\mathrm{q}$ are taken as 4 in the paper.

Data from January 1, 2018 to December 31, 2018 in Beijing were used as training data, and data from January 1, 2019 to January 31, 2019 were used as test data. Through the rolling prediction method, the data of the four days before the prediction day were used as input variables to predict the PM2.5 concentration values on that day. As shown in Table 1, a total of 44 factors are included in the obtained training data.

The LASSO method was applied to the training data for variable filtering, and the LASSO compressed path diagram is shown in Fig. 3. The colored dashed lines indicate the route of the factor selection process, the horizontal coordinates represent the constraint coefficients, and the vertical coordinates represent the regression coefficients. From Appendix A (Table A.1), it can be seen that Lasso proceeds to the 38th step with the smallest $c p$ value, and then the optimal number of steps for Lasso regression is 38 . Eight factors with non-zero coefficients were retained, as shown in the part marked with an asterisk in Table 1. The selected factors are those that have significant explanations for PM2.5 concentrations in Beijing. The above output shows that PM2.5 concentration in Beijing is influenced by its historical concentration, $\mathrm{SO} 2$ concentration, $\mathrm{CO}$ concentration, average temperature, wind speed and wind direction.

\section{Table 1}

The statement of influencing factors of PM2.5 concentration in Beijing

\begin{tabular}{ccccc}
\hline Name(Codename) & \multicolumn{5}{c}{ Lags } \\
\hline Daily PM2.5 concentration $\left(Y_{t}\right)$ & $Y_{t-1} *$ & $Y_{t-2}$ & $Y_{t-3}$ & $Y_{t-4}$ \\
Daily PM10 concentration $\left(X_{t}^{1}\right)$ & $X_{t-1}^{1}$ & $X_{t-2}^{1}$ & $X_{t-3}^{1}$ & $X_{t-4}^{1}$ \\
Daily SO2 concentration $\left(X_{t}^{2}\right)$ & $X_{t-1}^{2} *$ & $X_{t-2}^{2}$ & $X_{t-3}^{2}$ & $X_{t-4}^{2}$ \\
Daily CO concentration $\left(X_{t}^{3}\right)$ & $X_{t-1}^{3} *$ & $X_{t-2}^{3}$ & $X_{t-3}^{3} *$ & $X_{t-4}^{3}$ \\
Daily NO2 concentration $\left(X_{t}^{4}\right)$ & $X_{t-1}^{4}$ & $X_{t-2}^{4}$ & $X_{t-3}^{4}$ & $X_{t-4}^{4}$ \\
Daily O3 concentration $\left(X_{t}^{5}\right)$ & $X_{t-1}^{5}$ & $X_{t-2}^{5}$ & $X_{t-3}^{5}$ & $X_{t-4}^{5}$ \\
\hline
\end{tabular}




\begin{tabular}{rllll}
\hline Daily average temperature $\left(X_{t}^{6}\right)$ & $X_{t-1}^{6}$ & $X_{t-2}^{6} *$ & $X_{t-3}^{6} *$ & $X_{t-4}^{6}$ \\
Daily relative humidity $\left(X_{t}^{7}\right)$ & $X_{t-1}^{7}$ & $X_{t-2}^{7}$ & $X_{t-3}^{7}$ & $X_{t-4}^{7}$ \\
Daily average air pressure $\left(X_{t}^{8}\right)$ & $X_{t-1}^{8}$ & $X_{t-2}^{8}$ & $X_{t-3}^{8}$ & $X_{t-4}^{8}$ \\
Daily average wind speed $\left(X_{t}^{9}\right)$ & $X_{t-1}^{9} *$ & $X_{t-2}^{9}$ & $X_{t-3}^{9}$ & $X_{t-4}^{9}$ \\
The wind direction of daily maximum wind speed $\left(X_{t}^{10}\right)$ & $X_{t-1}^{10} *$ & $X_{t-2}^{10}$ & $X_{t-3}^{10}$ & $X_{t-4}^{10}$ \\
\hline
\end{tabular}

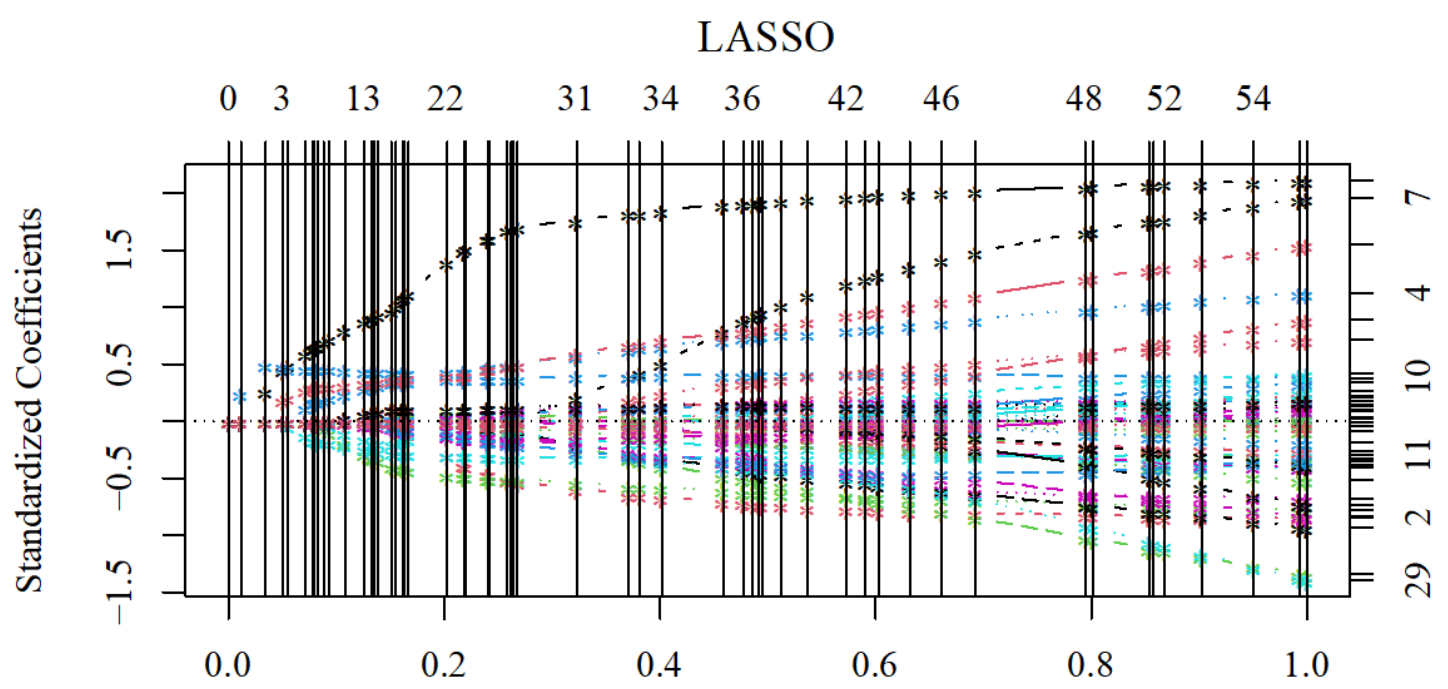

399

400

Fig.3. The procedure chart of compressing factors(Beijing)

Three situations are considered in the paper to make the empirical analysis more objective and to verify the validity of the LASSO-QR-KDE method for probability density prediction. They are probability density prediction without considering external factors, probability density prediction with considering external factors but without variable selection, and probability density prediction with applying LASSO for variable selection. After training, the neural network structures for the three cases are shown in Table 2. The number of times the neural network was trained was set to 1000, and the penalty parameters $\lambda_{1}$ and $\lambda_{2}$ in Eq. (19) were set to 0.5 . The quartiles range from 0.05 to 0.95 with an interval of 0.05 . With the trained neural network model, the continuous quartiles for each day in January 2019 can be obtained. The obtained PM2.5 concentration quantile is then used as an input variable and imported into the Epanechnikov kernel function to obtain the probability density curve of PM2.5 concentration for each day in January 2019.

Table 2 


\begin{tabular}{cc}
\hline Situation (Codename) & Input layer- Hidden layer- Output layer \\
\hline without considering external factors (A) & $7-3-1$ \\
considering external factors without variable selection (B) & $53-3-1$ \\
LASSO-QR-KDE(C) & $10-3-1$ \\
\hline
\end{tabular}

Fig. 4, Fig.5, and Fig.6 show the probability density curves of PM2.5 concentrations in Beijing for the solid blue line is the probability density curve and the highest point is the mode of the predicted value. The comparison of the three plots shows that the highest probability point (highest point) of the probability density curve often deviates from the actual value when no external factors are considered.

On days 1, 17, and 25 in Fig. 4, the actual values appear at the tail of the probability density curve, and the predicted values deviate significantly from the actual values. With external factors taken into account but not selected, the prediction situation improved. According to Figure 5, the prediction value that deviates significantly from the actual value is significantly improved, and only on day 13 the actual value is at the tail of the probability density curve. In the case of using LASSO to select the variables, according to Figure 6, the actual values are basically near the highest probability point of the probability density curve, and there is no case that the actual values are at the tail of the probability density curve. It can be seen that the LASSO-QR-KDE model has a good performance in predicting the probability density of PM2.5 concentration, and the predicted values have a higher probability of being close to the actual values. 

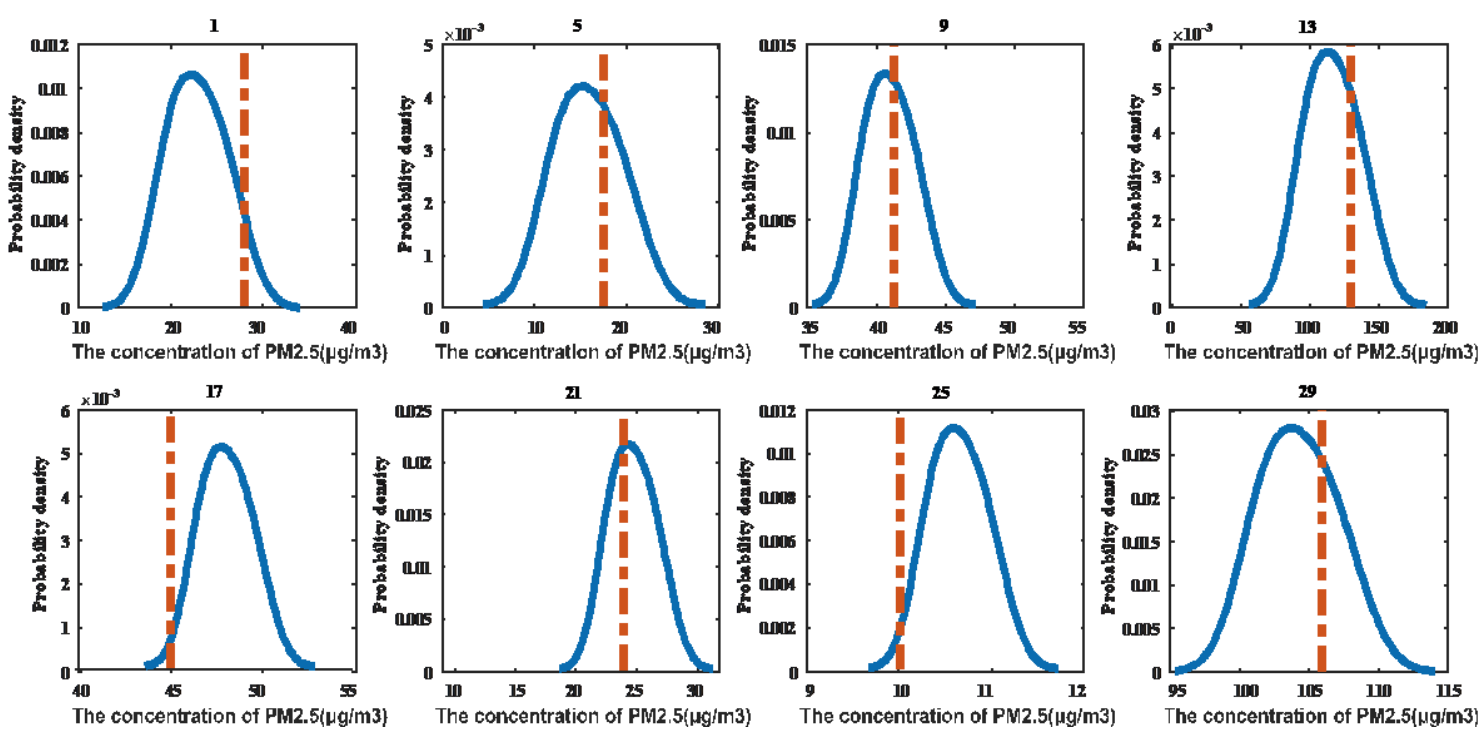

Fig.4. Probability density curve of Beijing without considering external factors
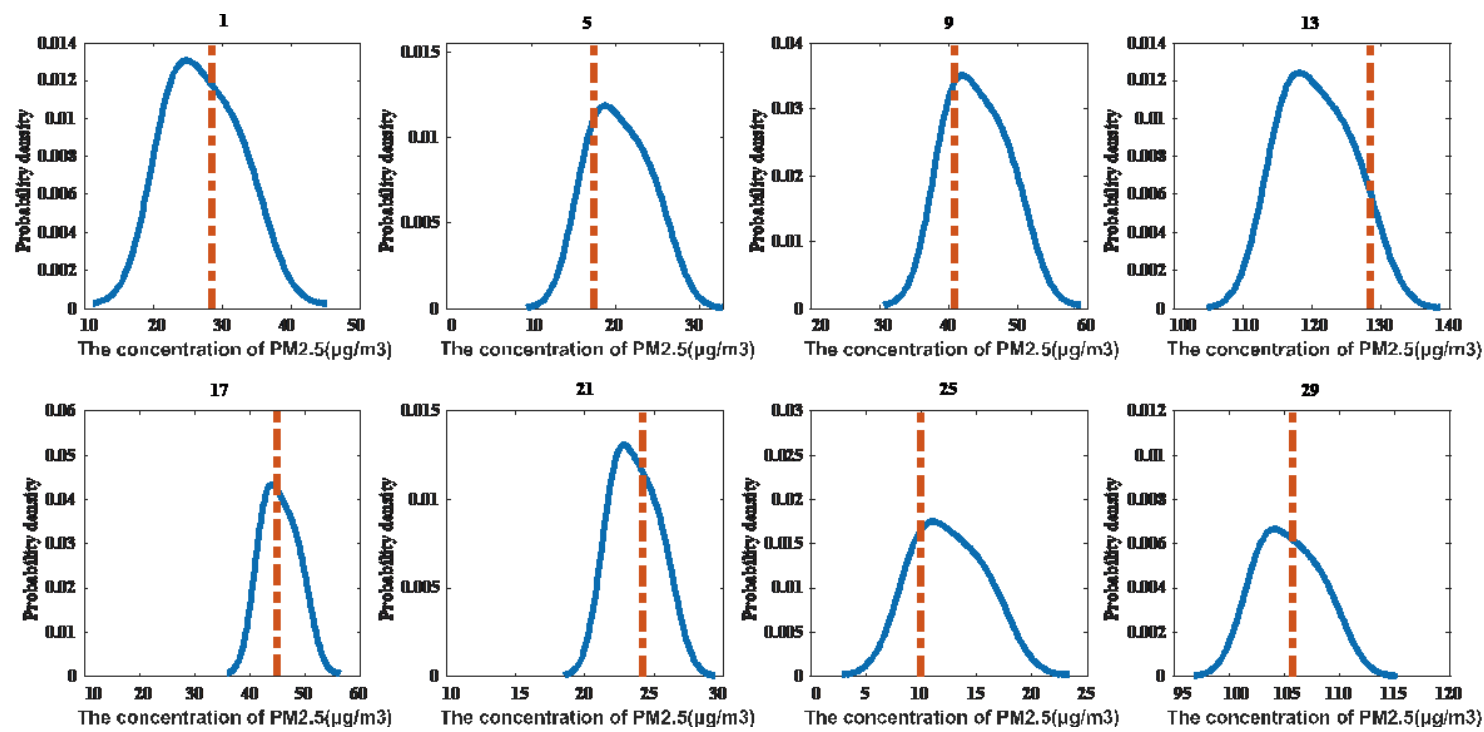

Fig.5. Probability density curve of Beijing considering external factors but without variable 

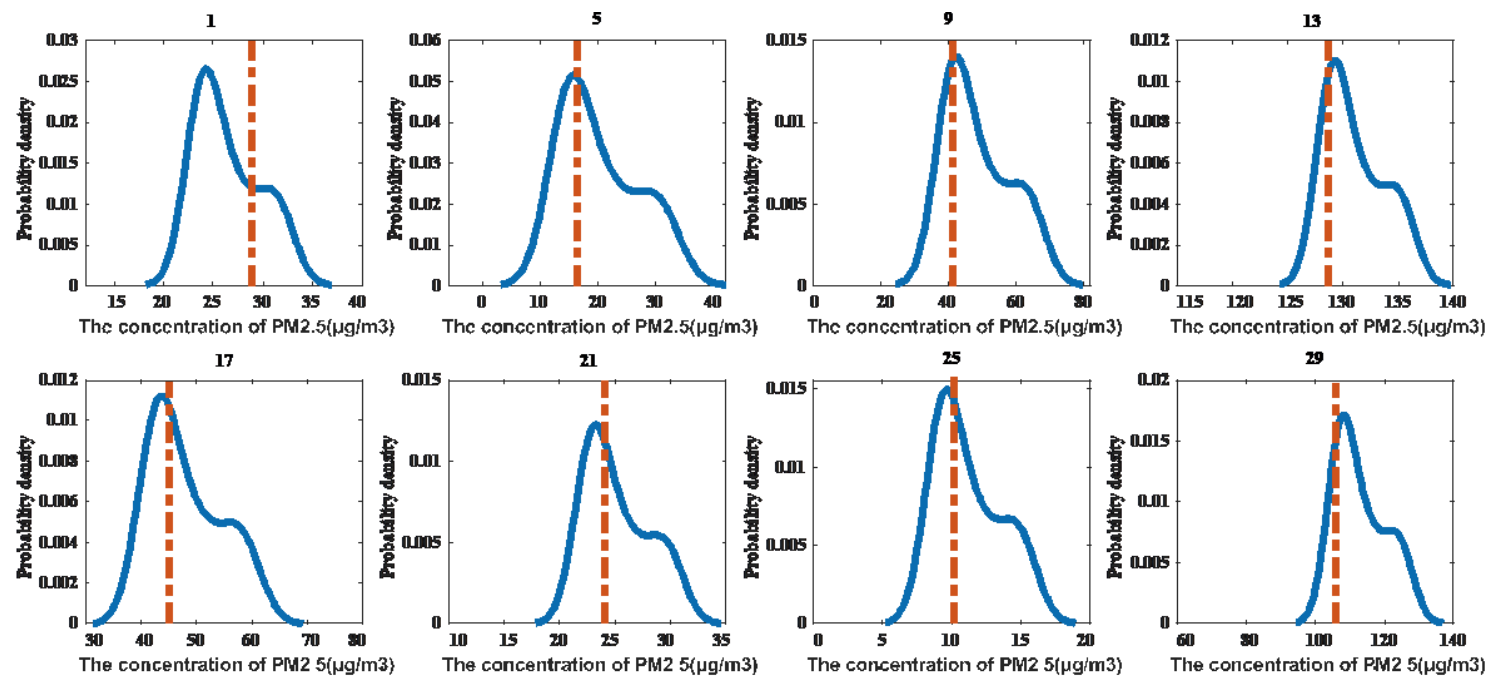

444

Fig.6. Probability density curve of Beijing with LASSO-QR-KDE model

In the prediction of PM2.5 concentrations with quantile regression neural networks, the prediction

results using the LASSO-QR-KDE model outperformed the prediction results without considering

external factors and without variable selection. This indicates that the probability density prediction of

PM2.5 concentration should adequately consider the influence of external environment, and the optimal

prediction model can be obtained by filtering multiple external factors.

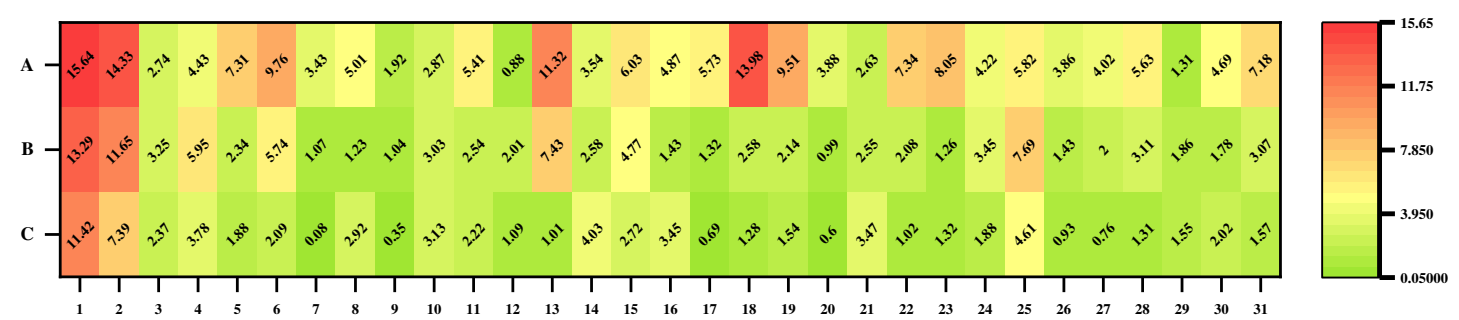

Fig. 7. Relative error of prediction results for three situations in Beijing (\%)

Table 3

Prediction errors for three situations in Beijing

\begin{tabular}{ccc}
\hline Situation (Codename) & MAPE(\%) & RMSE \\
\hline without considering external factors (A) & 6.04 & 4.11 \\
considering external factors without variable selection (B) & 3.41 & 2.74 \\
LASSO-QR-KDE(C) & 2.40 & 1.63 \\
\hline
\end{tabular}

\subsection{Case 2: Jinan, China}

Jinan is located in East China and has built 8 national automatic air environment quality 


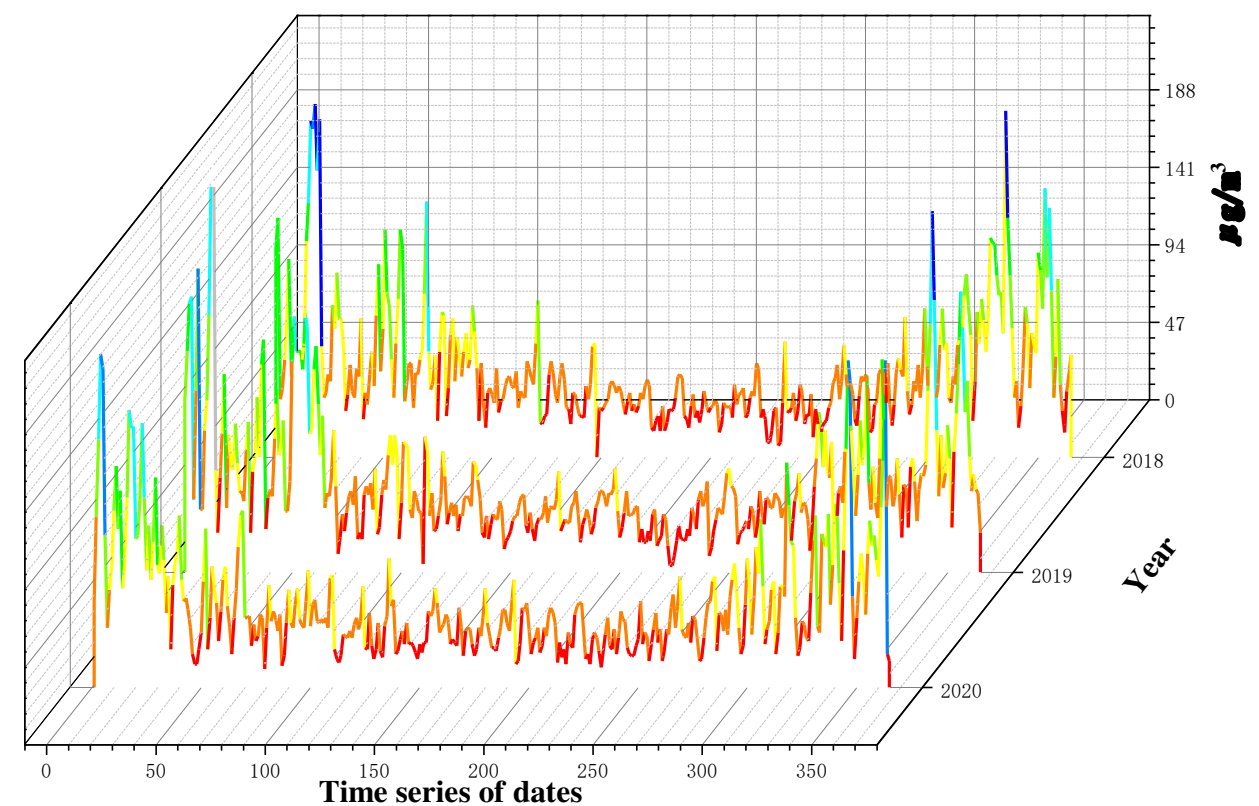

Fig.8. The PM2.5 concentration in Jinan from 2018 to 2020

In this case, data from January 1, 2020 to January 31, 2021 were selected from the National 
that the PM2.5 concentration in Jinan is influenced by its historical concentration, PM10 concentration,

\section{Table 4}

477 The statement of influencing factors of PM2.5 concentration in Jinan

\begin{tabular}{ccccc}
\hline Name(Codename) & \multicolumn{5}{c}{ Lags } \\
\hline Daily PM2.5 concentration $\left(Y_{t}\right)$ & $Y_{t-1} *$ & $Y_{t-2}$ & $Y_{t-3}$ & $Y_{t-4}$ \\
Daily PM10 concentration $\left(X_{t}^{1}\right)$ & $X_{t-1}^{1} *$ & $X_{t-2}^{1}$ & $X_{t-3}^{1}$ & $X_{t-4}^{1}$ \\
Daily SO2 concentration $\left(X_{t}^{2}\right)$ & $X_{t-1}^{2}$ & $X_{t-2}^{2}$ & $X_{t-3}^{2}$ & $X_{t-4}^{2}$ \\
Daily CO concentration $\left(X_{t}^{3}\right)$ & $X_{t-1}^{3} *$ & $X_{t-2}^{3} *$ & $X_{t-3}^{3} *$ & $X_{t-4}^{3}$ \\
Daily NO2 concentration $\left(X_{t}^{4}\right)$ & $X_{t-1}^{4} *$ & $X_{t-2}^{4}$ & $X_{t-3}^{4}$ & $X_{t-4}^{4}$ \\
Daily O3 concentration $\left(X_{t}^{5}\right)$ & $X_{t-1}^{5}$ & $X_{t-2}^{5}$ & $X_{t-3}^{5} *$ & $X_{t-4}^{5}$ \\
Daily average temperature $\left(X_{t}^{6}\right)$ & $X_{t-1}^{6}$ & $X_{t-2}^{6}$ & $X_{t-3}^{6}$ & $X_{t-4}^{6}$ \\
Daily relative humidity $\left(X_{t}^{7}\right)$ & $X_{t-1}^{7}$ & $X_{t-2}^{7}$ & $X_{t-3}^{7}$ & $X_{t-4}^{7}$ \\
Daily average air pressure $\left(X_{t}^{8}\right)$ & $X_{t-1}^{8}$ & $X_{t-2}^{8}$ & $X_{t-3}^{8}$ & $X_{t-4}^{8}$ \\
Daily average wind speed $\left(X_{t}^{9}\right)$ & $X_{t-1}^{9} *$ & $X_{t-2}^{9}$ & $X_{t-3}^{9} *$ & $X_{t-4}^{9}$ \\
The wind direction of daily maximum wind speed $\left(X_{t}^{10}\right)$ & $X_{t-1}^{10}$ & $X_{t-2}^{10} *$ & $X_{t-3}^{10} *$ & $X_{t-4}^{10}$ \\
\hline
\end{tabular}

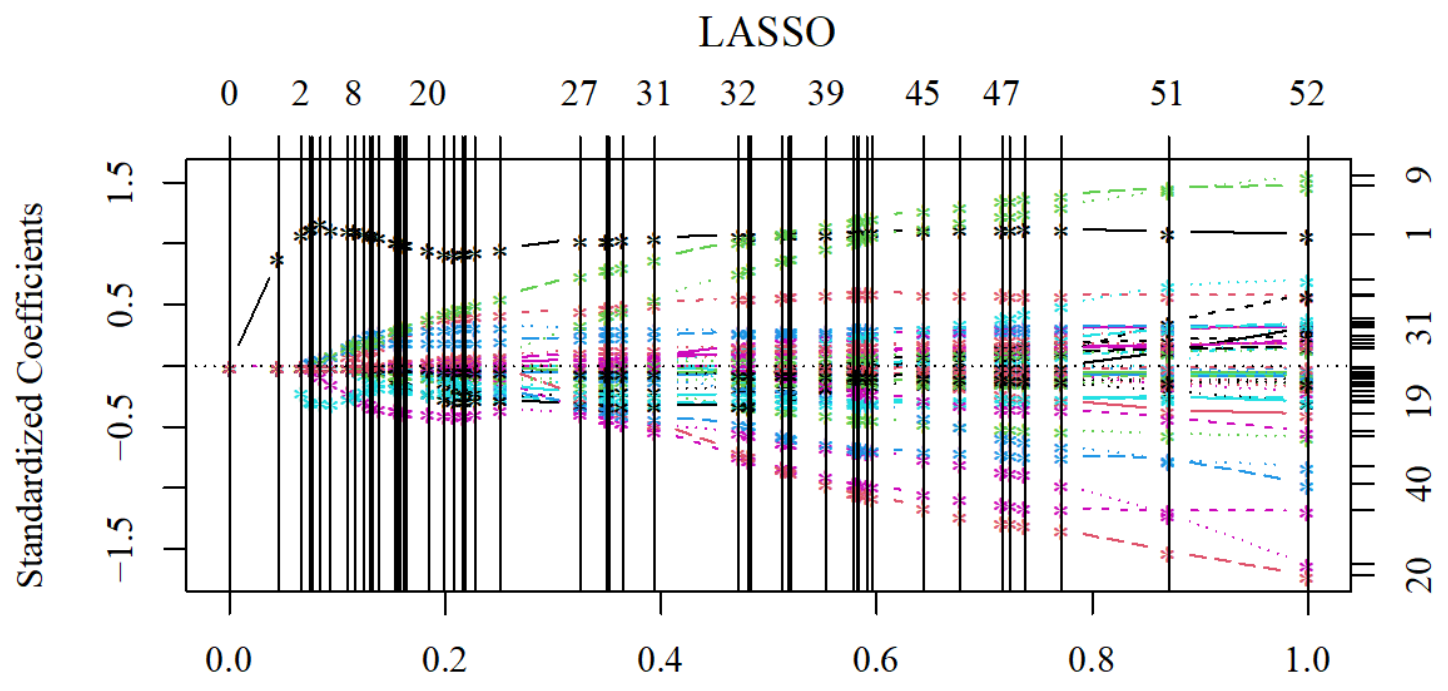

Fig.9. The procedure chart of compressing factors(Jinan) 
Structure of neural network in three situations(Jinan)

\begin{tabular}{cc}
\hline Situation (Codename) & Input layer- Hidden layer- Output layer \\
\hline without considering external factors (A) & $6-5-1$ \\
considering external factors without variable selection (B) & $40-5-1$ \\
LASSO-QR-KDE(C) & $12-5-1$ \\
\hline
\end{tabular}

Fig. 10, Fig. 11, and Fig. 12 show the probability density curves of PM2.5 concentrations in Jinan
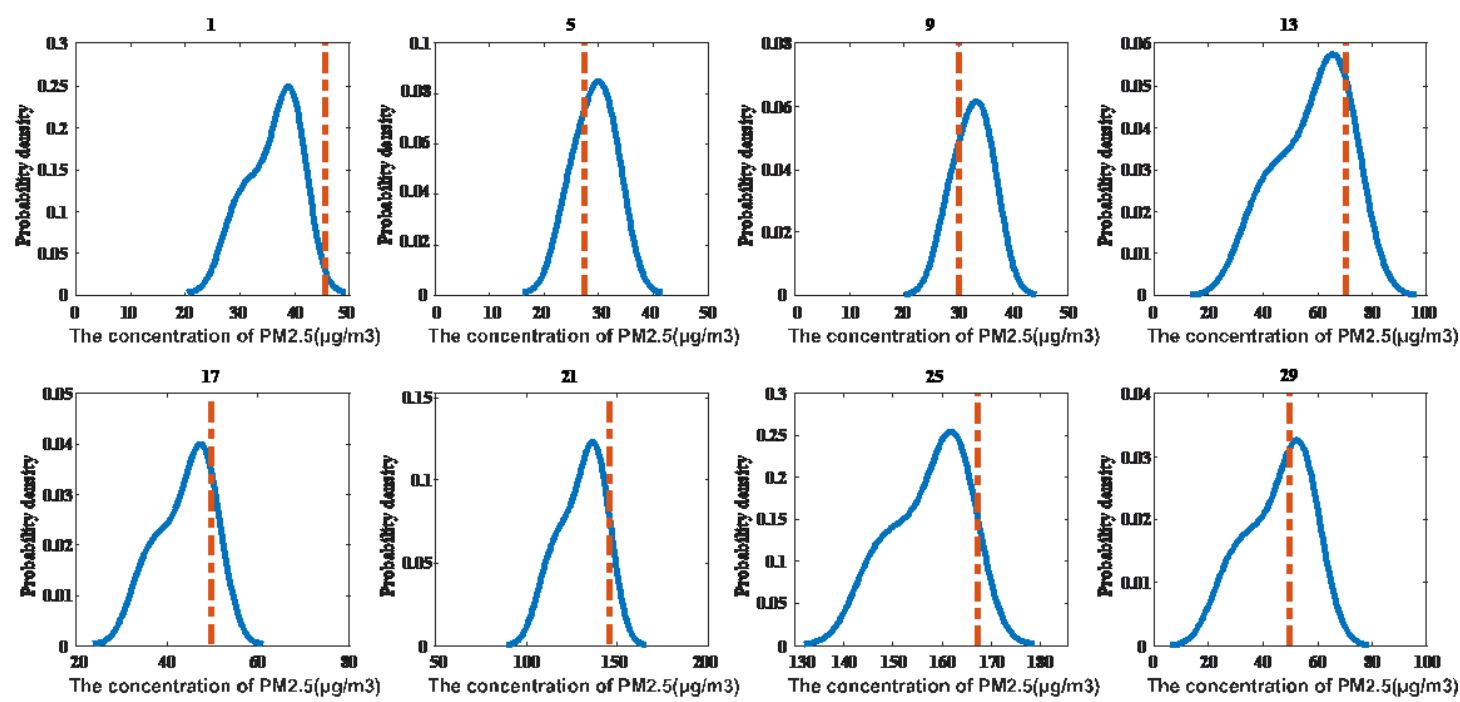

Fig.10. Probability density curve of Jinan without considering external factors 

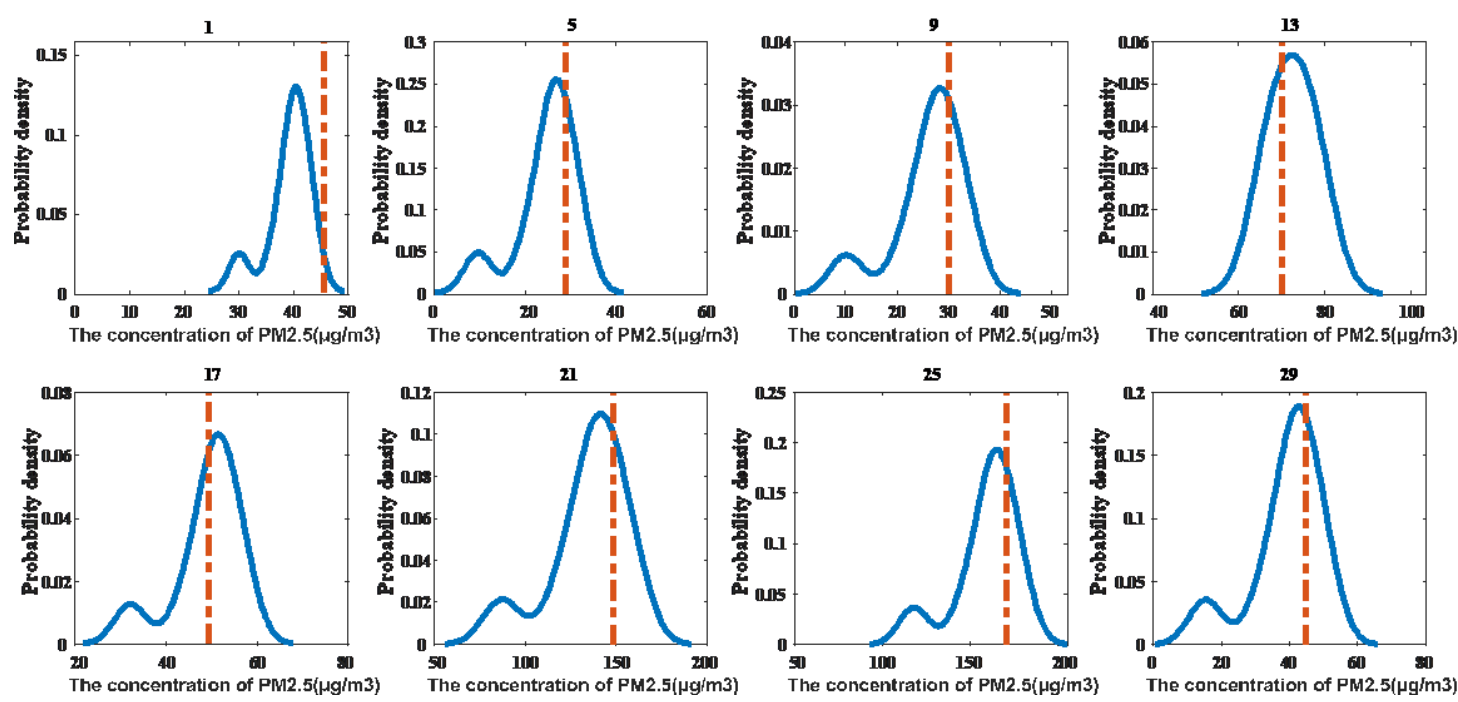

496

497

Fig.11. Probability density curve of Jinan considering external factors but without variable selection
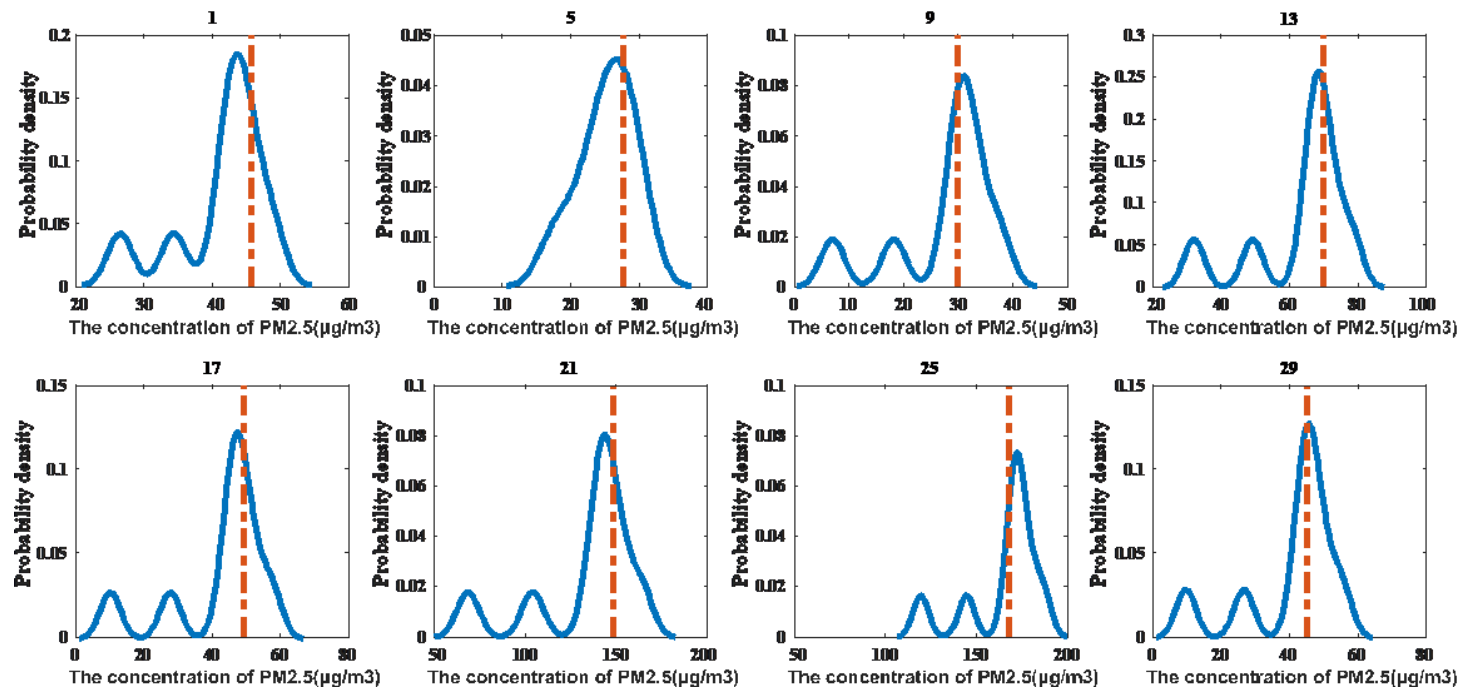

Fig.12. Probability density curve of Beijing with LASSO-QR-KDE model

Appendix B (Table B.2) presents the predicted values of PM2.5 concentrations at the highest point of the probability density curve (mode) and the relative errors for Jinan in January 2021 under three situations. Fig. 13 shows that the maximum relative errors in the three cases are $14.77 \%, 11.46 \%$ and $6.52 \%$, and the minimum relative errors are $1.02 \%, 1.53 \%$ and $0.05 \%$. The overall performance using the LASSO-QR-KDE model is significantly improved over the other two situations. The experimental results in Table 6 show that the mode has the best prediction results when applying LASSO for variable selection, where MAPE is $2.36 \%$ and RMSE is 2.07 . Similar to Case 1 , probability density prediction 
of PM2.5 concentration using LASSO-QR-KDE model can get more accurate results.

508

509

510

511

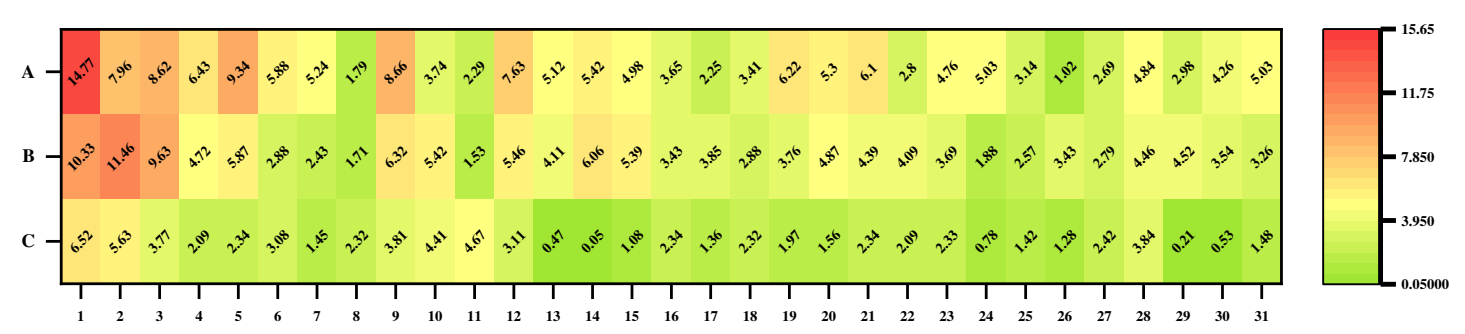

Fig. 13. Relative error of prediction results for three situations in Jinan (\%)

\section{Table 6}

Prediction errors for three situations in Jinan

\begin{tabular}{ccc}
\hline Situation (Codename) & MAPE(\%) & RMSE \\
\hline without considering external factors (A) & 5.20 & 4.74 \\
considering external factors without variable selection (B) & 4.54 & 4.50 \\
LASSO-QR-KDE(C) & 2.36 & 2.07 \\
\hline
\end{tabular}

\subsection{Comparisons with other models}

To demonstrate the superiority of the LASSO-QR-KDE method for PM2.5 concentration prediction, a variety of traditional prediction models and novel research models are introduced in this section for comparison with the proposed method. In this section, the prediction results are compared using the same data set. Five comparison models are presented below.

(1) ARIMA (Autoregressive Integrated Moving Average model), which achieves time series forecasting by fitting the observed time series into a parametric model (Ruby-Figueroa et al. 2016);

(2) BP (Back Propagation Neural Network), which continuously adjusts the weights and thresholds of the network through training, and thus learns the mapping relationships in the input and output data (Zhou and Qu 2017);

(3) RBF (Radical Basis Function), similar to BP, but with good ability to approximate arbitrary nonlinear functions and to express the intrinsic unresolved regularities of the system (He et al. 2013);

(4) CNN-LSTM (convolutional neural networks-long short termmemory ), which uses CNN for 
feature extraction, and then inputs the extracted feature values into the LSTM structure (Huang and Kuo 2018).

(5) EMD-GRU (Empirical Mode Decomposition- Gate Recurrent Unit), which uses EMD to decompose PM2.5 concentration sequences, and then the decomposed multiple smooth subsequences and meteorological features are sequentially input into the GRU neural network for training and prediction (Huang et al. 2021).

The prediction results of each model for PM2.5 concentrations in Beijing are shown in Fig. 14 and for Jinan City in Fig. 15. The prediction errors of the two cases are compared in Fig. 16. In addition, the daily forecast data for each model are shown in Appendix $\mathrm{C}$ along with the daily forecast relative errors.
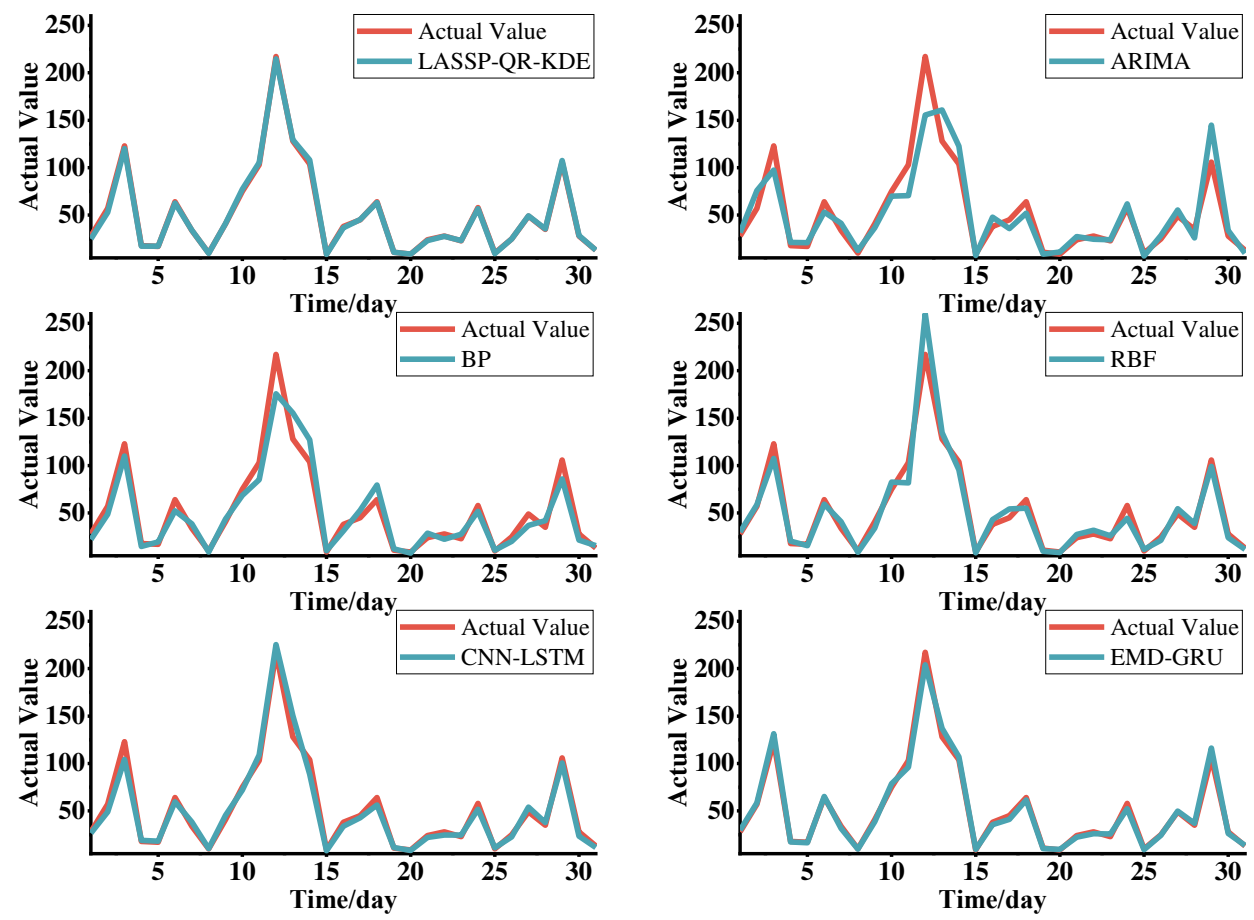

Fig. 14 The comparison between the predicted and actual values of each model (Beijing) 

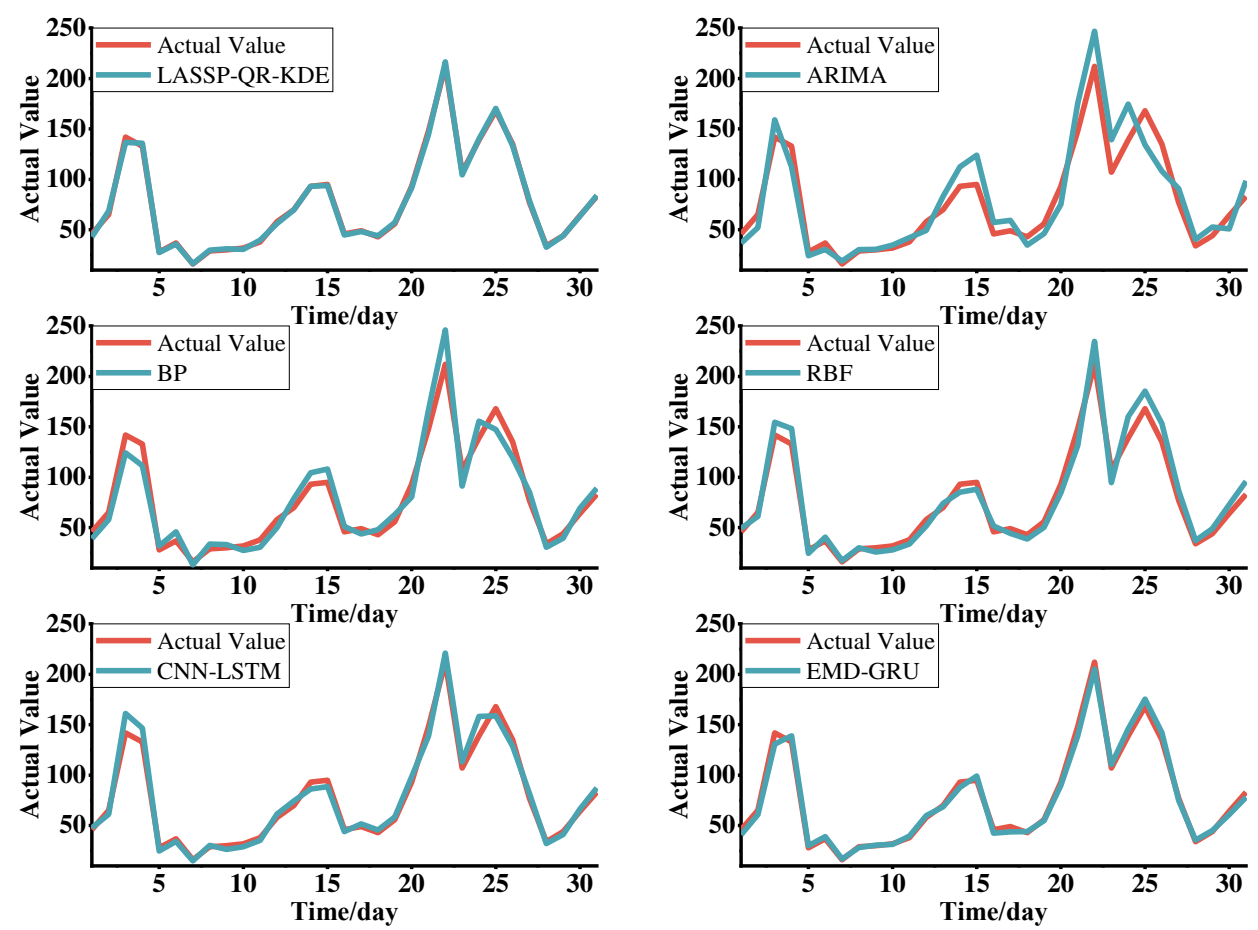

Fig. 15 The comparison between the predicted and actual values of each model (Jinan)
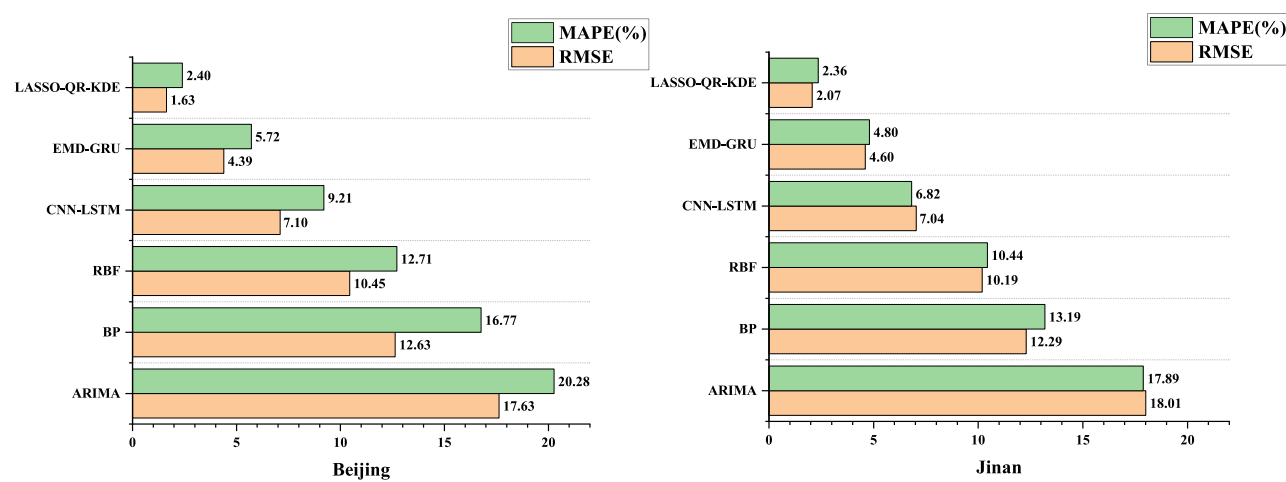

Fig. 16 Prediction error for each model

Based on the experimental results of each model, the following observations are derived:

(1) Compared with the traditional prediction model ARIMA and the classical machine learning

methods BP and RBF, the evaluation indexes of the other three models are better, which reflects that 
single model.

548

549

550

551

552

553

554

555

556

557

558

559

560

561

(2) In both cases, the RMSE and MAPE of the LASSO-QR-KDE model are taken to be the minimum values. Taking the data of the Beijing case as an example, the RMSE and MAPE of the proposed model decreased by 2.14 and $2.78 \%$, respectively, compared to the better-performing EMD-GRU model.

\subsection{Discussion}

In this paper, a new hybrid model is proposed to accurately predict the short-term variation of PM2.5 concentrations. Based on previous studies, LASSO is used for the selection of factors influencing PM2.5 concentrations, which greatly improves the prediction accuracy of the model. Similar to the findings of Koen et al.(2021), the LASSO method does contribute to a larger effect in improving the accuracy of the model interpretation. In addition, due to the specific nature of quantile regression, the effect of each factor on PM2.5 concentrations can be explained at different quantile points. The QR model provides the overall distribution of the response variables corresponding to the explanatory variables, which gives more information than the widely used mean regression (Sun et al. 2021). After obtaining the predicted values of the daily PM2.5 concentrations at multiple quartiles, the KDE model was used to estimate the probability density function of the daily PM2.5 concentrations, and the value corresponding to the highest point of the probability density curve was the predicted value of the concentration .

In summary, compared with other commonly used prediction models, the LASSO-QR-KDE model proposed in the paper can effectively identify the sequences that are significant to the prediction target and can obtain the probability distribution of the predicted values. Both cases involved in the 
paper are areas with poor air quality and high PM2.5 concentrations, however, their main causes are different. Pollution from industrial development is a major factor in the poor air quality in Beijing, China, and in Jinan, China, it is the deposition of air pollutants due to its topography. The LASSO-QR-KDE model is able to perform better in the prediction results of both cases, indicating that the method is suitable for the prediction of areas with high PM2.5 concentrations. In future research, extension of the model to prediction of PM2.5 concentrations in general could be considered, and better selection methods for influence factors are also a future research direction.

\section{Conclusion}

This paper proposes the LASSO-QR-KDE model to predict the probability density of PM2.5 concentrations. Based on two cases in the Chinese region, the important influencing factors of PM2.5 concentrations are firstly screened out, and then the data are processed using the proposed model. The LASSO-QR-KDE model is applied to obtain continuous probability density prediction curves, which can provide more information for PM2.5 concentration prediction. According to the empirical analysis, the main findings are as follows:

(1) The LASSO-QR-KDE model starts from the perspective of dealing with high-dimensional data and is able to select factors that have a significant impact on PM2.5 concentrations. The model can eliminate irrelevant variables and achieve the goal of effectively reducing the data dimensionality without affecting the prediction performance. This paper compares the probability density prediction without considering external factors, the probability density prediction with considering external factors but without variable screening, and the probability density prediction with variable screening using LASSO. We found that the prediction of PM2.5 concentration is significantly affected by 
considering external factors or not. The LASSO-QR-KDE model can enhance the model prediction accuracy effectively when external factors are considered.

(2) Applying the LASSO-QR-KDE model to PM2.5 concentration data can obtain the probability density prediction curve for each consecutive day in a month, and the true value almost appears near the highest point of the probability density curve. The continuous probability density curve can provide more information for the prediction of PM2.5 concentration, and can obtain more abundant prediction results compared with other prediction methods.

(3) From the experimental results of the two cases in the paper, there are differences in the important factors affecting PM2.5 concentrations in different regions. The RMSE and MAPE of both cases can be maintained around 2 and $2 \%$, which indicates the applicability and accuracy of the method for prediction in areas with high PM2.5 concentrations. The model can effectively identify important features from the high-dimensional data and make differentiated predictions for areas with high PM2.5 concentrations, which can provide meaningful references for relevant policy makers to effectively reduce and control air pollution and reduce public health risks.

\section{CRediT authorship contribution statement}

Shaomei Yang: Methodology, Resources, Writing - review \& editing.

Haoyue Wu: Conceptualization, Software, Validation, Inves-tigation, Data curation, Writing original draft.

\section{Data availability statement}

The datasets analysed during the current study are available in the the National Meteorological 
Ethical Approval

611 Not applicable.

612

613

614

615

616

617

618

619

620

621

622

623

624

625

626

627

628

\section{Consent to Participate}

Not applicable.

\section{Consent to Publish}

Not applicable.

\section{Funding}

This research did not receive any specific grant from funding agencies in the public, commercial, or not-for-profit sectors.

\section{Declaration of competing interest}

The authors declare that they have no known competing financial interests or personal relationships that could have appeared to influence the work reported in this paper.

\section{References}

Breiman, Leo. Better Subset Regression Using the Nonnegative Garrote[J]. Technometrics, 1995, 37(4):373-384.

Cao M N,Tian P,Li G R. Study on Lasso Penalized Quantile Regression of Optimal Portfolio [J/OL]. Journal of Systems Science and Mathematical Sciences:1-17[2021-06-19].

Cao Q, Lian S, Chen S C, et al. WRF modeling of PM2.5 remediation by SALSCS and its clean air flow over Beijing terrain[J]. Science of the Total Environment, 2018, 626(JUN.1):134. 
Geng G, Zhang Q, Martin R V, et al. Estimating long-term PM2.5, concentrations in China using satellite-based aerosol optical depth and a chemical transport model[J]. Remote Sensing of Environment, 2015, 166: 262-270.

He P,Lan W,Ding Y. Is the Chinese Stock Market Predictable?-An Evidence Based on the Combination LASSO-logistic Model [J]. Statistical Research,2021,38(05):82-96.

He Y Y, Xu Q F, Yang S L, et al. A power load probability density forecasting method based on RBF neural network quantile Regression[J]. Zhongguo Dianji Gongcheng Xuebao/Proceedings of the Chinese Society of Electrical Engineering, 2013, 33(1):93-98.

He Y Y, Yang Q, Wang S, et al. Electricity consumption probability density forecasting method based on LASSO-Quantile Regression Neural Network[J]. Applied Energy, 2019, s 233 234(JAN.1):565-575.

Hoerl A , Kennard R . Taylor \& Francis Online :: Ridge Regression: Biased Estimation for Nonorthogonal Problems - Technometrics - Volume 12, Issue 1[J]. Technometrics, 2000, 42(1):7.

Huang C J , Kuo P H . A Deep CNN-LSTM Model for Particulate Matter (PM2.5) Forecasting in Smart Cities[J]. Sensors, 2018, 18(7):2220.

Huang G, Li X, Zhang B, et al. PM2. 5 concentration forecasting at surface monitoring sites using GRU neural network based on empirical mode decomposition[J]. Science of The Total Environment, 2021, 768: 144516.

Jasleen Kaur Sethi,Mamta Mittal. An efficient correlation based adaptive LASSO regression method for air quality index prediction[J]. Earth Science Informatics,2021(prepublish).

Jiang F,Qiao Y Q. PM2.5 Concentration Prediction Based on Sample Entropy and Improved Extreme Learning Machine [J]. Statistics \& Decision,2021,37(03):166-171. 
651

652

653

654

655

656

657

658

659

660

661

662

663

664

665

666

667

668

669

670

671

672

Kang Qiaoling,Wu Ji,Chen Minghua,Jeon Bang Nam. Do macroprudential policies affect the bank financing of firms in China? Evidence from a quantile regression approach[J]. Journal of International Money and Finance,2021,115.

Koenker R . Quantile Regression[J]. Journal of Economic Perspectives, 2015, 15(4):143-156.

Koen Füssenich, Boshuizen H C , Nielen M M J , et al. Mapping Chronic Disease Prevalence based on Medication Use and Socio-demographic variables: an Application of LASSO in healthcare in the Netherlands. 2020.

Lee D, Mukhopadhyay S, Rushworth A, et al. A rigorous statistical framework for spatio-temporal pollution prediction and estimation of its long-term impact on health[J]. Biostatistics, 2017, 18(2): $370-385$

Li Chunyu,Lou Chenxin,Luo Dan,Xing Kai. Chinese corporate distress prediction using LASSO:

The role of earnings management[J]. International Review of Financial Analysis,2021(prepublish).

Lightstone S D , Moshary F , Gross B . Comparing CMAQ Forecasts with a Neural Network Forecast Model for PM2.5 in New York[J]. Atmosphere, 2017, 8(12):161-.

Li W, Kong D , Wu J . A New Hybrid Model FPA-SVM Considering Cointegration for Particular Matter Concentration Forecasting: A Case Study of Kunming and Yuxi, China[J]. Computational Intelligence and Neuroscience,2017,(2017-8-28), 2017, 2017:1-11.

Madsen K , Nielsen H B . A Finite Smoothing Algorithm for Linear \$1_1 \$ Estimation[J]. Siam Journal on Optimization, 1993, 3(2):223-235.

Mukhopadhyay S, Sahu S K. A Bayesian spatiotemporal model to estimate long-term exposure to outdoor air pollution at coarser administrative geographies in England and Wales[J]. Journal of the Royal Statistical Society (Series A)- Statistics in Society, 2018, 181(2): 465-486. 
674 Squared-Error versus Absolute-Error Estimators[J]. Statistical Science, 1997, 12(4).

ultrafiltration of fruit juices by ARIMA models[J]. Journal of Membrane Science, 2016:S0376738816307207.

Salari Taghi Ebrahimi,Roumiani Ahmad,Kazemzadeh Emad. Globalization, renewable energy consumption, and agricultural production impacts on ecological footprint in emerging countries: using quantile regression approach.[J]. Environmental science and pollution research international,2021.

Schwarz G E . Estimating the Dimension of a Model[J]. The Annals of Statistics, 1978, 6(2).

Sheather S J , Jones M C . A reliable data-based bandwidth selection method for kernel density estimation[J]. Journal of the Royal Statistical Society. Series B: Methodological, 1991, 53(3):683-690.

Sun Jie,Zhao Xiaojun,Xu Chao. Crude oil market autocorrelation: Evidence from multiscale quantile regression analysis[J]. Energy Economics,2021,98.

Syrakov D, Prodanova M , Georgieva E , et al. Simulation of European air quality by WRFCMAQ models using AQMEII-2 infrastructure[J]. Journal of Computational \& Applied Mathematics, 2016:232-245.

Taha Alshaybawee,Rahim Alhamzawi,Habshah Midi,Intisar Ibrahim Allyas. Bayesian variable selection and coefficient estimation in heteroscedastic linear regression model[J]. Journal of Applied Statistics,2018,45(14).

Tibshirani R . Regression shrinkage and selection via the lasso[J]. Journal of the Royal Statistical Society, Series B, 1996, 58(1). 
700 algorithm to prediction of air quality index in Lanzhou[C]// 2017 2nd IEEE International Conference

701 on Computational Intelligence and Applications (ICCIA). IEEE, 2017.

Table A.1

705 LASSO regression results of Beijing data

\begin{tabular}{rrrrrrrrrrrl}
\hline Step & Df & \multicolumn{1}{l}{ Rss } & \multicolumn{1}{c}{ Cp } & Step & Df & Rss & Cp & Step & Df & Rss & Cp \\
\hline 0 & 1 & 4.76 & 419.17 & 19 & 16 & 2.24 & 70.00 & $38^{*}$ & 31 & 1.83 & 37.67 \\
1 & 2 & 4.18 & 333.85 & 20 & 17 & 2.24 & 71.06 & 39 & 32 & 1.83 & 39.35 \\
2 & 3 & 3.33 & 208.06 & 21 & 18 & 2.23 & 71.49 & 40 & 33 & 1.82 & 39.81 \\
3 & 4 & 3.00 & 160.10 & 22 & 19 & 2.14 & 60.01 & 41 & 34 & 1.80 & 39.78 \\
4 & 5 & 2.94 & 152.46 & 23 & 20 & 2.11 & 57.53 & 42 & 35 & 1.79 & 39.09 \\
5 & 6 & 2.74 & 125.38 & 24 & 21 & 2.10 & 59.03 & 43 & 36 & 1.78 & 39.93 \\
6 & 7 & 2.68 & 118.01 & 25 & 22 & 2.07 & 55.93 & 44 & 37 & 1.77 & 41.10 \\
7 & 8 & 2.68 & 118.95 & 26 & 21 & 2.07 & 53.53 & 45 & 38 & 1.76 & 41.31 \\
8 & 9 & 2.65 & 117.39 & 27 & 22 & 2.05 & 52.10 & 46 & 39 & 1.75 & 41.62 \\
9 & 10 & 2.65 & 118.72 & 28 & 23 & 2.04 & 53.22 & 47 & 40 & 1.74 & 42.04 \\
10 & 9 & 2.60 & 110.15 & 29 & 24 & 2.04 & 55.05 & 48 & 41 & 1.71 & 40.00 \\
11 & 10 & 2.57 & 106.98 & 30 & 25 & 2.03 & 56.14 & 49 & 42 & 1.71 & 41.79 \\
12 & 11 & 2.47 & 94.52 & 31 & 26 & 1.97 & 48.49 & 50 & 41 & 1.70 & 38.48 \\
13 & 12 & 2.38 & 82.09 & 32 & 27 & 1.92 & 43.32 & 51 & 42 & 1.70 & 40.39 \\
14 & 11 & 2.35 & 75.60 & 33 & 28 & 1.91 & 43.94 & 52 & 43 & 1.70 & 42.19 \\
15 & 12 & 2.34 & 76.18 & 34 & 29 & 1.89 & 43.30 & 38 & 31 & 1.83 & 37.67 \\
16 & 13 & 2.32 & 75.94 & 35 & 30 & 1.85 & 38.86 & 39 & 32 & 1.83 & 39.35 \\
17 & 14 & 2.28 & 70.92 & 36 & 31 & 1.84 & 38.96 & 40 & 33 & 1.82 & 39.81 \\
18 & 15 & 2.26 & 70.80 & 37 & 32 & 1.83 & 40.20 & 41 & 34 & 1.80 & 39.78 \\
\hline & & & & & & & & & & &
\end{tabular}


LASSO regression results of Jinan data

\begin{tabular}{rrrrrrrrrrrr}
\hline Step & Df & Rss & \multicolumn{1}{l}{ Cp } & Step & Df & \multicolumn{1}{l}{ Rss } & Cp & Step & Df & Rss & Cp \\
\hline 0 & 1 & 6.82 & 465.33 & 19 & 18 & 2.88 & 57.72 & 38 & 33 & 2.37 & 30.58 \\
1 & 2 & 4.36 & 192.16 & 20 & 19 & 2.82 & 53.35 & 39 & 34 & 2.35 & 30.61 \\
2 & 3 & 3.70 & 120.33 & 21 & 20 & 2.78 & 51.48 & 40 & 35 & 2.34 & 31.34 \\
3 & 4 & 3.53 & 102.75 & 22 & 21 & 2.76 & 51.16 & 41 & 36 & 2.34 & 33.23 \\
4 & 5 & 3.49 & 100.29 & 23 & 22 & 2.75 & 51.11 & 42 & 37 & 2.33 & 35.12 \\
5 & 6 & 3.35 & 86.65 & 24 & 23 & 2.74 & 52.50 & 43 & 38 & 2.33 & 36.81 \\
6 & 7 & 3.23 & 75.10 & 25 & 24 & 2.72 & 52.41 & 44 & 39 & 2.33 & 38.60 \\
7 & 8 & 3.10 & 62.32 & 26 & 25 & 2.68 & 49.27 & 45 & 40 & 2.31 & 38.85 \\
8 & 9 & 3.06 & 60.18 & 27 & 26 & 2.55 & 37.63 & 46 & 41 & 2.31 & 39.86 \\
9 & 10 & 3.05 & 61.65 & 28 & 27 & 2.52 & 36.02 & 47 & 40 & 2.30 & 36.98 \\
10 & 11 & 3.02 & 59.27 & 29 & 28 & 2.52 & 37.68 & 48 & 41 & 2.30 & 38.86 \\
11 & 12 & 2.99 & 58.89 & 30 & 29 & 2.50 & 37.87 & 49 & 42 & 2.29 & 40.63 \\
12 & 13 & 2.98 & 59.81 & 31 & 30 & 2.47 & 36.09 & 50 & 43 & 2.29 & 42.17 \\
13 & 14 & 2.96 & 59.14 & $32 *$ & 31 & 2.40 & 30.20 & 51 & 44 & 2.28 & 43.33 \\
14 & 15 & 2.91 & 55.42 & 33 & 32 & 2.39 & 31.45 & 52 & 45 & 2.28 & 45.00 \\
15 & 16 & 2.90 & 56.63 & 34 & 33 & 2.39 & 33.28 & 38 & 33 & 2.37 & 30.58 \\
16 & 17 & 2.89 & 57.62 & 35 & 34 & 2.37 & 33.14 & 39 & 34 & 2.35 & 30.61 \\
17 & 16 & 2.88 & 54.48 & 36 & 33 & 2.37 & 31.10 & 40 & 35 & 2.34 & 31.34 \\
18 & 17 & 2.88 & 55.97 & 37 & 34 & 2.37 & 32.69 & 41 & 36 & 2.34 & 33.23 \\
\hline
\end{tabular}

708

\section{Appendix B}

710

Table B.1

711 Prediction results for three situations of Beijing data

\begin{tabular}{|c|c|c|c|c|c|c|c|}
\hline \multirow{2}{*}{$\begin{array}{l}\text { Time } \\
\text { /day }\end{array}$} & \multirow{2}{*}{$\begin{array}{c}\text { Actual } \\
\text { value } \\
\left(\mu g / m^{3}\right)\end{array}$} & \multicolumn{2}{|c|}{ A } & \multicolumn{2}{|c|}{$\mathrm{B}$} & \multicolumn{2}{|c|}{$\mathrm{C}$} \\
\hline & & $\begin{array}{c}\text { Mode } \\
\left(\mu g / m^{3}\right)\end{array}$ & $\operatorname{RE}(\%)$ & $\begin{array}{c}\text { Mode } \\
\left(\mu g / m^{3}\right)\end{array}$ & $\operatorname{RE}(\%)$ & $\begin{array}{c}\text { Mode } \\
\left(\mu g / m^{3}\right)\end{array}$ & $\operatorname{RE}(\%)$ \\
\hline 1 & 28 & 23.62 & 15.64 & 24.28 & 13.29 & 24.80 & 11.42 \\
\hline 2 & 57 & 48.83 & 14.33 & 50.36 & 11.65 & 52.79 & 7.39 \\
\hline 3 & 123 & 119.63 & 2.74 & 119.00 & 3.25 & 120.08 & 2.37 \\
\hline 4 & 18 & 17.20 & 4.43 & 19.07 & 5.95 & 17.32 & 3.78 \\
\hline 5 & 17 & 15.76 & 7.31 & 17.40 & 2.34 & 16.68 & 1.88 \\
\hline 6 & 64 & 70.25 & 9.76 & 67.67 & 5.74 & 62.66 & 2.09 \\
\hline 7 & 34 & 35.17 & 3.43 & 33.64 & 1.07 & 34.03 & 0.08 \\
\hline 8 & 10 & 9.50 & 5.01 & 10.12 & 1.23 & 9.71 & 2.92 \\
\hline 9 & 41 & 40.21 & 1.92 & 41.43 & 1.04 & 41.14 & 0.35 \\
\hline 10 & 75 & 72.85 & 2.87 & 77.27 & 3.03 & 77.35 & 3.13 \\
\hline 11 & 103 & 108.57 & 5.41 & 100.38 & 2.54 & 105.29 & 2.22 \\
\hline 12 & 217 & 218.91 & 0.88 & 221.36 & 2.01 & 214.63 & 1.09 \\
\hline 13 & 128 & 113.51 & 11.32 & 118.49 & 7.43 & 129.29 & 1.01 \\
\hline
\end{tabular}




\begin{tabular}{cccccccc}
\hline $\mathbf{1 4}$ & 104 & 100.32 & 3.54 & 101.32 & 2.58 & 108.19 & 4.03 \\
$\mathbf{1 5}$ & 9 & 8.46 & 6.03 & 9.43 & 4.77 & 8.76 & 2.72 \\
$\mathbf{1 6}$ & 38 & 39.85 & 4.87 & 37.46 & 1.43 & 36.69 & 3.45 \\
$\mathbf{1 7}$ & 45 & 47.58 & 5.73 & 44.41 & 1.32 & 44.69 & 0.69 \\
$\mathbf{1 8}$ & 64 & 72.95 & 13.98 & 62.35 & 2.58 & 63.18 & 1.28 \\
$\mathbf{1 9}$ & 11 & 12.05 & 9.51 & 11.24 & 2.14 & 10.83 & 1.54 \\
$\mathbf{2 0}$ & 9 & 9.35 & 3.88 & 8.91 & 0.99 & 9.05 & 0.60 \\
$\mathbf{2 1}$ & 24 & 24.63 & 2.63 & 23.39 & 2.55 & 23.17 & 3.47 \\
$\mathbf{2 2}$ & 28 & 30.06 & 7.34 & 28.58 & 2.08 & 27.71 & 1.02 \\
$\mathbf{2 3}$ & 23 & 21.15 & 8.05 & 22.71 & 1.26 & 23.30 & 1.32 \\
$\mathbf{2 4}$ & 58 & 60.45 & 4.22 & 60.00 & 3.45 & 56.91 & 1.88 \\
$\mathbf{2 5}$ & 10 & 10.58 & 5.82 & 10.77 & 7.69 & 9.54 & 4.61 \\
$\mathbf{2 6}$ & 25 & 24.04 & 3.86 & 25.36 & 1.43 & 24.77 & 0.93 \\
$\mathbf{2 7}$ & 49 & 50.97 & 4.02 & 49.98 & 2.00 & 49.37 & 0.76 \\
$\mathbf{2 8}$ & 35 & 36.97 & 5.63 & 33.91 & 3.11 & 35.46 & 1.31 \\
$\mathbf{2 9}$ & 106 & 104.61 & 1.31 & 104.03 & 1.86 & 107.64 & 1.55 \\
$\mathbf{3 0}$ & 28 & 29.31 & 4.69 & 27.50 & 1.78 & 28.57 & 2.02 \\
$\mathbf{3 1}$ & 13 & 12.07 & 7.18 & 13.40 & 3.07 & 13.20 & 1.57 \\
\hline
\end{tabular}

\section{Table B.2}

713 Prediction results for three situations of Jinan data

\begin{tabular}{cccccccc}
\hline \multirow{2}{*}{$\begin{array}{c}\text { Time } \\
\text { dday }\end{array}$} & $\begin{array}{c}\text { Actual } \\
\text { value } \\
\left(\mu g / \mathrm{m}^{3}\right)\end{array}$ & $\begin{array}{c}\text { Mode } \\
\left(\mu g / \mathrm{m}^{3}\right)\end{array}$ & $\mathrm{RE}(\%)$ & $\begin{array}{c}\text { Mode } \\
\left(\mu g / \mathrm{m}^{3}\right)\end{array}$ & $\mathrm{RE}(\%)$ & $\begin{array}{c}\text { Mode } \\
\left(\mu g / \mathrm{m}^{3}\right)\end{array}$ & $\mathrm{RE}(\%)$ \\
\hline $\mathbf{1}$ & 46 & 39.21 & 14.77 & 41.25 & 10.33 & 43.00 & 6.52 \\
$\mathbf{2}$ & 65 & 70.17 & 7.96 & 72.45 & 11.46 & 68.66 & 5.63 \\
$\mathbf{3}$ & 142 & 129.76 & 8.62 & 128.33 & 9.63 & 136.65 & 3.77 \\
$\mathbf{4}$ & 133 & 141.55 & 6.43 & 139.28 & 4.72 & 135.78 & 2.09 \\
$\mathbf{5}$ & 28 & 30.62 & 9.34 & 26.36 & 5.87 & 27.34 & 2.34 \\
$\mathbf{6}$ & 37 & 39.18 & 5.88 & 35.93 & 2.88 & 35.86 & 3.08 \\
$\mathbf{7}$ & 16 & 15.16 & 5.24 & 16.39 & 2.43 & 16.23 & 1.45 \\
$\mathbf{8}$ & 29 & 28.48 & 1.79 & 28.50 & 1.71 & 29.67 & 2.32 \\
$\mathbf{9}$ & 30 & 32.60 & 8.66 & 28.10 & 6.32 & 31.14 & 3.81 \\
$\mathbf{1 0}$ & 32 & 33.20 & 3.74 & 33.73 & 5.42 & 30.59 & 4.41 \\
$\mathbf{1 1}$ & 38 & 37.13 & 2.29 & 38.58 & 1.53 & 39.77 & 4.67 \\
$\mathbf{1 2}$ & 58 & 53.57 & 7.63 & 61.17 & 5.46 & 56.20 & 3.11 \\
$\mathbf{1 3}$ & 70 & 66.42 & 5.12 & 72.88 & 4.11 & 69.67 & 0.47 \\
$\mathbf{1 4}$ & 93 & 98.04 & 5.42 & 87.36 & 6.06 & 93.05 & 0.05 \\
$\mathbf{1 5}$ & 95 & 90.27 & 4.98 & 100.12 & 5.39 & 93.97 & 1.08 \\
$\mathbf{1 6}$ & 46 & 47.68 & 3.65 & 44.42 & 3.43 & 44.92 & 2.34 \\
$\mathbf{1 7}$ & 49 & 47.90 & 2.25 & 50.89 & 3.85 & 48.33 & 1.36 \\
$\mathbf{1 8}$ & 43 & 41.53 & 3.41 & 44.24 & 2.88 & 44.00 & 2.32 \\
$\mathbf{1 9}$ & 56 & 59.48 & 6.22 & 53.89 & 3.76 & 57.10 & 1.97 \\
$\mathbf{2 0}$ & 93 & 97.93 & 5.3 & 88.47 & 4.87 & 91.55 & 1.56 \\
\hline
\end{tabular}




\begin{tabular}{lccccccc}
\hline $\mathbf{2 1}$ & 148 & 138.97 & 6.1 & 141.50 & 4.39 & 144.54 & 2.34 \\
$\mathbf{2 2}$ & 212 & 217.94 & 2.8 & 220.67 & 4.09 & 216.43 & 2.09 \\
$\mathbf{2 3}$ & 107 & 101.91 & 4.76 & 103.05 & 3.69 & 104.51 & 2.33 \\
$\mathbf{2 4}$ & 139 & 132.01 & 5.03 & 141.61 & 1.88 & 140.08 & 0.78 \\
$\mathbf{2 5}$ & 168 & 162.72 & 3.14 & 163.68 & 2.57 & 170.39 & 1.42 \\
$\mathbf{2 6}$ & 135 & 133.62 & 1.02 & 130.37 & 3.43 & 133.27 & 1.28 \\
$\mathbf{2 7}$ & 77 & 79.07 & 2.69 & 79.15 & 2.79 & 78.86 & 2.42 \\
$\mathbf{2 8}$ & 34 & 32.35 & 4.84 & 35.52 & 4.46 & 32.69 & 3.84 \\
$\mathbf{2 9}$ & 44 & 45.31 & 2.98 & 42.01 & 4.52 & 44.09 & 0.21 \\
$\mathbf{3 0}$ & 64 & 61.27 & 4.26 & 61.73 & 3.54 & 63.66 & 0.53 \\
$\mathbf{3 1}$ & 83 & 87.17 & 5.03 & 85.71 & 3.26 & 84.23 & 1.48 \\
\hline
\end{tabular}

714

Appendix C

715 Table C.1.1

716 Predicted values under various models of Beijing data $\left(\mu \mathrm{g} / \mathrm{m}^{3}\right)$

\begin{tabular}{|c|c|c|c|c|c|c|c|}
\hline Time/day & $\begin{array}{l}\text { Actual } \\
\text { value }\end{array}$ & LASSO-QR-KDE & ARIMA & $\mathrm{BP}$ & $\mathrm{RBF}$ & CNN-LSTM & EMD-GRU \\
\hline 1 & 24.80 & 31.66 & 22.15 & 30.08 & 26.57 & 29.58 & 24.80 \\
\hline 2 & 52.79 & 75.88 & 48.76 & 58.70 & 49.06 & 58.42 & 52.79 \\
\hline 3 & 120.08 & 97.35 & 109.97 & 107.47 & 104.16 & 131.36 & 120.08 \\
\hline 4 & 17.32 & 21.32 & 14.88 & 20.40 & 18.90 & 17.30 & 17.32 \\
\hline 5 & 17.32 & 21.04 & 19.62 & 15.56 & 18.27 & 16.35 & 17.32 \\
\hline 6 & 62.66 & 53.18 & 52.24 & 59.35 & 59.56 & 65.24 & 62.66 \\
\hline 7 & 34.03 & 41.57 & 38.51 & 40.83 & 38.47 & 31.69 & 34.03 \\
\hline 8 & 9.71 & 12.92 & 8.99 & 8.66 & 10.36 & 9.66 & 9.71 \\
\hline 9 & 41.14 & 36.59 & 43.71 & 34.10 & 45.35 & 38.58 & 41.14 \\
\hline 10 & 77.35 & 70.12 & 68.60 & 82.55 & 71.96 & 78.59 & 77.35 \\
\hline 11 & 105.29 & 70.62 & 85.16 & 81.75 & 109.08 & 95.95 & 105.29 \\
\hline 12 & 214.63 & 155.48 & 175.70 & 259.99 & 225.22 & 204.08 & 214.63 \\
\hline 13 & 129.29 & 160.92 & 155.42 & 134.66 & 150.35 & 137.06 & 129.29 \\
\hline 14 & 108.19 & 122.76 & 127.12 & 95.41 & 88.65 & 107.02 & 108.19 \\
\hline 15 & 8.76 & 7.43 & 10.42 & 7.97 & 8.19 & 9.45 & 8.76 \\
\hline 16 & 36.69 & 47.78 & 30.97 & 43.21 & 33.74 & 35.42 & 36.69 \\
\hline 17 & 45.31 & 35.75 & 53.01 & 54.40 & 42.92 & 41.12 & 45.31 \\
\hline 18 & 63.18 & 52.06 & 79.40 & 55.17 & 56.25 & 61.60 & 63.18 \\
\hline 19 & 10.83 & 8.86 & 12.37 & 9.83 & 11.52 & 10.26 & 10.83 \\
\hline 20 & 9.05 & 11.12 & 7.93 & 8.24 & 8.50 & 9.42 & 9.05 \\
\hline 21 & 23.17 & 27.51 & 28.76 & 27.50 & 22.04 & 22.41 & 23.17 \\
\hline 22 & 27.71 & 24.72 & 22.25 & 31.82 & 24.50 & 25.91 & 27.71 \\
\hline 23 & 23.30 & 24.15 & 27.58 & 25.93 & 24.51 & 25.61 & 23.30 \\
\hline 24 & 56.91 & 62.00 & 51.74 & 44.56 & 51.85 & 52.41 & 56.91 \\
\hline 25 & 9.54 & 6.97 & 10.76 & 11.64 & 11.10 & 9.14 & 9.54 \\
\hline 26 & 24.77 & 28.46 & 20.14 & 21.17 & 22.60 & 24.42 & 24.77 \\
\hline
\end{tabular}




\begin{tabular}{cccccccc}
\hline 27 & 49.37 & 55.32 & 37.00 & 54.63 & 54.08 & 49.73 & 49.37 \\
28 & 35.46 & 26.02 & 42.02 & 38.84 & 37.89 & 37.04 & 35.46 \\
29 & 107.64 & 144.94 & 85.87 & 99.27 & 100.62 & 116.27 & 107.64 \\
30 & 28.57 & 33.98 & 21.60 & 24.02 & 23.36 & 26.26 & 28.57 \\
31 & 13.20 & 9.65 & 15.53 & 11.72 & 11.36 & 13.55 & 13.20 \\
\hline
\end{tabular}

Table C.1.2

718 Relative errors under various models of Beijing data (\%)

\begin{tabular}{|c|c|c|c|c|c|c|}
\hline Time/day & LASSO-QR-KDE & ARIMA & $\mathrm{BP}$ & $\mathrm{RBF}$ & CNN-LSTM & EMD-GRU \\
\hline 1 & 11.42 & 13.07 & 20.88 & 7.42 & 5.11 & 5.63 \\
\hline 2 & 7.39 & 33.12 & 14.46 & 2.98 & 13.93 & 2.49 \\
\hline 3 & 2.37 & 20.85 & 10.59 & 12.63 & 15.32 & 6.80 \\
\hline 4 & 3.78 & 18.45 & 17.32 & 13.31 & 4.99 & 3.89 \\
\hline 5 & 1.88 & 23.77 & 15.44 & 8.48 & 7.46 & 3.80 \\
\hline 6 & 2.09 & 16.90 & 18.38 & 7.26 & 6.93 & 1.94 \\
\hline 7 & 0.08 & 22.25 & 13.25 & 20.09 & 13.14 & 6.80 \\
\hline 8 & 2.92 & 29.24 & 10.09 & 13.42 & 3.55 & 3.38 \\
\hline 9 & 0.35 & 10.76 & 6.60 & 16.84 & 10.62 & 5.91 \\
\hline 10 & 3.13 & 6.51 & 8.54 & 10.06 & 4.06 & 4.78 \\
\hline 11 & 2.22 & 31.44 & 17.32 & 20.63 & 5.90 & 6.84 \\
\hline 12 & 1.09 & 28.35 & 19.03 & 19.81 & 3.79 & 5.95 \\
\hline 13 & 1.01 & 25.72 & 21.42 & 5.20 & 17.46 & 7.08 \\
\hline 14 & 4.03 & 18.04 & 22.23 & 8.26 & 14.76 & 2.91 \\
\hline 15 & 2.72 & 17.40 & 15.76 & 11.45 & 9.01 & 5.02 \\
\hline 16 & 3.45 & 25.73 & 18.50 & 13.70 & 11.20 & 6.80 \\
\hline 17 & 0.69 & 20.56 & 17.79 & 20.88 & 4.62 & 8.63 \\
\hline 18 & 1.28 & 18.66 & 24.07 & 13.79 & 12.11 & 3.75 \\
\hline 19 & 1.54 & 19.42 & 12.43 & 10.66 & 4.69 & 6.75 \\
\hline 20 & 0.60 & 23.58 & 11.86 & 8.48 & 5.53 & 4.66 \\
\hline 21 & 3.47 & 14.62 & 19.84 & 14.57 & 8.18 & 6.61 \\
\hline 22 & 1.02 & 11.73 & 20.53 & 13.64 & 12.49 & 7.45 \\
\hline 23 & 1.32 & 5.02 & 19.93 & 12.75 & 6.59 & 11.36 \\
\hline 24 & 1.88 & 6.89 & 10.80 & 23.18 & 10.60 & 9.64 \\
\hline 25 & 4.61 & 30.33 & 7.63 & 16.37 & 10.98 & 8.63 \\
\hline 26 & 0.93 & 13.82 & 19.45 & 15.32 & 9.61 & 2.33 \\
\hline 27 & 0.76 & 12.90 & 24.48 & 11.48 & 10.37 & 1.49 \\
\hline 28 & 1.31 & 25.67 & 20.05 & 10.97 & 8.25 & 5.83 \\
\hline 29 & 1.55 & 36.74 & 18.99 & 6.35 & 5.07 & 9.69 \\
\hline 30 & 2.02 & 21.36 & 22.85 & 14.22 & 16.59 & 6.21 \\
\hline 31 & 1.57 & 25.79 & 19.47 & 9.81 & 12.64 & 4.23 \\
\hline
\end{tabular}

719 Table C.2.1

720 Predicted values under various models of Jinan data $\left(\mu \mathrm{g} / \mathrm{m}^{3}\right)$

\begin{tabular}{llllllll}
\hline Time/day & Actual & LASSO-QR-KDE & ARIMA & BP & RBF & CNN-LSTM & EMD-GRU \\
\hline
\end{tabular}




\begin{tabular}{|c|c|c|c|c|c|c|c|}
\hline & value & & & & & & \\
\hline 1 & 46 & 43.00 & 36.65 & 38.90 & 49.37 & 47.60 & 40.88 \\
\hline 2 & 65 & 68.66 & 52.15 & 57.93 & 61.16 & 61.17 & 60.94 \\
\hline 3 & 142 & 136.65 & 159.07 & 124.32 & 154.55 & 161.06 & 130.78 \\
\hline 4 & 133 & 135.78 & 112.45 & 111.48 & 148.07 & 146.57 & 138.88 \\
\hline 5 & 28 & 27.34 & 24.25 & 31.82 & 24.63 & 24.79 & 30.06 \\
\hline 6 & 37 & 35.86 & 30.47 & 45.90 & 40.46 & 34.17 & 39.12 \\
\hline 7 & 16 & 16.23 & 18.90 & 13.24 & 17.37 & 15.09 & 16.78 \\
\hline 8 & 29 & 29.67 & 30.28 & 33.81 & 30.13 & 30.40 & 28.10 \\
\hline 9 & 30 & 31.14 & 30.46 & 33.26 & 25.81 & 26.23 & 30.65 \\
\hline 10 & 32 & 30.59 & 34.81 & 27.29 & 27.93 & 29.09 & 31.28 \\
\hline 11 & 38 & 39.77 & 42.14 & 30.66 & 33.62 & 35.20 & 39.42 \\
\hline 12 & 58 & 56.20 & 49.18 & 50.19 & 51.67 & 61.47 & 59.93 \\
\hline 13 & 70 & 69.67 & 83.12 & 78.88 & 74.07 & 74.29 & 68.56 \\
\hline 14 & 93 & 93.05 & 112.21 & 104.38 & 85.14 & 86.26 & 87.89 \\
\hline 15 & 95 & 93.97 & 123.91 & 108.10 & 88.16 & 88.51 & 99.05 \\
\hline 16 & 46 & 44.92 & 57.08 & 51.20 & 51.79 & 44.04 & 42.37 \\
\hline 17 & 49 & 48.33 & 59.23 & 43.63 & 44.36 & 51.76 & 43.84 \\
\hline 18 & 43 & 44.00 & 34.77 & 48.04 & 38.67 & 45.67 & 44.00 \\
\hline 19 & 56 & 57.10 & 46.13 & 62.96 & 50.47 & 58.42 & 54.84 \\
\hline 20 & 93 & 91.55 & 75.11 & 80.31 & 84.65 & 98.33 & 89.80 \\
\hline 21 & 148 & 144.54 & 175.25 & 166.57 & 131.22 & 138.75 & 138.90 \\
\hline 22 & 212 & 216.43 & 246.75 & 246.13 & 234.75 & 220.88 & 205.09 \\
\hline 23 & 107 & 104.51 & 139.12 & 91.24 & 94.71 & 113.23 & 110.57 \\
\hline 24 & 139 & 140.08 & 174.56 & 155.64 & 160.00 & 158.11 & 145.70 \\
\hline 25 & 168 & 170.39 & 134.22 & 147.49 & 185.34 & 158.73 & 175.41 \\
\hline 26 & 135 & 133.27 & 108.04 & 119.41 & 153.13 & 128.74 & 142.24 \\
\hline 27 & 77 & 78.86 & 90.75 & 85.26 & 86.36 & 81.71 & 74.77 \\
\hline 28 & 34 & 32.69 & 40.29 & 30.67 & 37.19 & 32.09 & 35.71 \\
\hline 29 & 44 & 44.09 & 52.79 & 39.60 & 49.02 & 41.10 & 45.02 \\
\hline 30 & 64 & 63.66 & 50.85 & 69.27 & 72.86 & 66.39 & 60.81 \\
\hline 31 & 83 & 84.23 & 98.54 & 89.08 & 95.97 & 87.17 & 78.01 \\
\hline
\end{tabular}

Table C.2.2

722 Relative errors under various models of Jinan data (\%)

\begin{tabular}{ccccccc}
\hline Time/day & LASSO-QR-KDE & ARIMA & BP & RBF & CNN-LSTM & EMD-GRU \\
\hline 1 & 6.52 & 20.32 & 15.44 & 7.33 & 3.47 & 11.14 \\
2 & 5.63 & 19.77 & 10.87 & 5.91 & 5.89 & 6.25 \\
3 & 3.77 & 12.02 & 12.45 & 8.84 & 13.42 & 7.90 \\
4 & 2.09 & 15.45 & 16.18 & 11.33 & 10.20 & 4.42 \\
5 & 2.34 & 13.38 & 13.64 & 12.05 & 11.45 & 7.34 \\
6 & 3.08 & 17.64 & 24.06 & 9.36 & 7.66 & 5.72 \\
7 & 1.45 & 18.15 & 17.23 & 8.57 & 5.71 & 4.85 \\
8 & 2.32 & 4.43 & 16.59 & 3.88 & 4.83 & 3.09 \\
\hline
\end{tabular}




\begin{tabular}{ccccccc}
\hline 9 & 3.81 & 1.54 & 10.88 & 13.97 & 12.56 & 2.16 \\
10 & 4.41 & 8.77 & 14.71 & 12.72 & 9.10 & 2.25 \\
11 & 4.67 & 10.90 & 19.32 & 11.53 & 7.36 & 3.74 \\
12 & 3.11 & 15.20 & 13.46 & 10.91 & 5.99 & 3.33 \\
13 & 0.47 & 18.74 & 12.69 & 5.82 & 6.13 & 2.06 \\
14 & 0.05 & 20.66 & 12.24 & 8.45 & 7.25 & 5.50 \\
15 & 1.08 & 30.43 & 13.79 & 7.20 & 6.83 & 4.26 \\
16 & 2.34 & 24.09 & 11.30 & 12.58 & 4.27 & 7.89 \\
17 & 1.36 & 20.88 & 10.96 & 9.46 & 5.63 & 10.53 \\
18 & 2.32 & 19.15 & 11.73 & 10.08 & 6.20 & 2.32 \\
19 & 1.97 & 17.63 & 12.42 & 9.87 & 4.33 & 2.08 \\
20 & 1.56 & 19.24 & 13.64 & 8.98 & 5.73 & 3.44 \\
21 & 2.34 & 18.41 & 12.55 & 11.34 & 6.25 & 6.15 \\
22 & 2.09 & 16.39 & 16.10 & 10.73 & 4.19 & 3.26 \\
23 & 2.33 & 30.02 & 14.73 & 11.49 & 5.82 & 3.34 \\
24 & 0.78 & 25.58 & 11.97 & 15.11 & 13.75 & 4.82 \\
25 & 1.42 & 20.11 & 12.21 & 10.32 & 5.52 & 4.41 \\
26 & 1.28 & 19.97 & 11.55 & 13.43 & 4.64 & 5.36 \\
27 & 2.42 & 17.86 & 10.73 & 12.15 & 6.12 & 2.90 \\
28 & 3.84 & 18.49 & 9.79 & 9.39 & 5.62 & 5.04 \\
29 & 0.21 & 19.98 & 10.01 & 11.42 & 6.60 & 2.31 \\
30 & 0.53 & 20.54 & 8.24 & 13.84 & 3.74 & 4.99 \\
31 & 1.48 & 18.72 & 7.32 & 15.63 & 5.03 & 6.01 \\
\hline & & & & & &
\end{tabular}

